\title{
BV EXPONENTIAL STABILITY FOR SYSTEMS OF SCALAR CONSERVATION LAWS USING SATURATED CONTROLS
}

\author{
MATHIAS DUS*
}

\begin{abstract}
In this paper, we investigate the $B V$ exponential stability of general systems of scalar conservation laws with positive velocities and under dissipative boundary conditions. The paper is divided in two parts, the first one focusing on linear controls while the last one deals with saturated laws. For the linear case, the global exponential $B V$ stability is proved. For the saturated case, it is discussed that we cannot expect to have a basin of attraction larger than the region of linearity in a $B V$ context. We rather prove an $L^{\infty}$ local stability result. An explicit estimate of the basin of attraction is given. The Lyapunov functional is inspired from Glimm's seminal work [18] reconsidered in [9].
\end{abstract}

Key words. Bounded variations, stabilization, feedback, saturation, wavefront tracking method.

AMS subject classifications. 93D05, 93D15, 93D20

1. Introduction. In this work, the focus is on the exponential stabilization of some 1D hyperbolic systems using saturated feedback control laws. More precisely, we are interested in systems of $d \in \mathbb{N}$ scalar conservation laws with strictly positive characteristic velocities. The system under consideration is of the form:

$$
\forall i \in \llbracket 1, d \rrbracket, \begin{cases}R_{i, t}+\left[f_{i}\left(R_{i}\right)\right]_{x} & =0 \\ R_{i}(t, 0) & =g_{i}(R(t, 1)) \\ R_{i}(0, x) & =R_{0, i}(x)\end{cases}
$$

where $R_{i}: \mathbb{R}^{+} \times[0,1] \mapsto \mathbb{R}, f_{i}: \mathbb{R} \mapsto \mathbb{R}$ and $g_{i}: \mathbb{R}^{d} \mapsto \mathbb{R}$. For coherence, all characteristic velocities are positive and consequently, the boundary condition in (1.1) is adapted. More specifically, we are interested in the stabilization of (1.1) using feedback control laws at the boundary. The problem is equivalent to find sufficient conditions on $g$ such that for any initial data $R_{0}$, the solution to (1.1) converges exponentially fast towards zero in the sense that

$$
\forall t \geq 0,\|R(t, .)\|_{X} \leq C e^{-\gamma t}\left\|R_{0}\right\|_{X}
$$

where $C, \gamma>0$ are constants independent on $t$ and $\|\cdot\|_{X}$ is a norm on a Banach space $X$.

1.1. An example. One can consider the basic scalar model for open channel [3, p.44]:

$$
\partial_{t} R+\partial_{x}(k R \sqrt{R})=0
$$

where $R>0$ is the height of water in the channel, $k$ is a coefficient calculated from the viscous friction, the vertical slope of the channel and the gravity. This simplified model corresponds to a regime where the friction is compensated by the gravity. Written in the flow rate variable $Q=k R^{3 / 2}>0^{1},(1.3)$ writes:

${ }^{*}$ Univ. Paul Sabatier, Institut de Mathématiques de Toulouse, 118 route de Narbonne, 31062 Toulouse Cedex 9 (mathias.dus@math.univ-toulouse.fr).

${ }^{1}$ Here the change of variable is done when the solution is regular. With discontinuous solutions, equations (1.3) and (1.4) may not be equivalent. 


$$
\partial_{t} Q+\frac{9}{8} k^{2 / 3} \partial_{x}\left(Q^{4 / 3}\right)=0
$$

Now imagine that seven channels are linked as depicted in Figure 1 ;

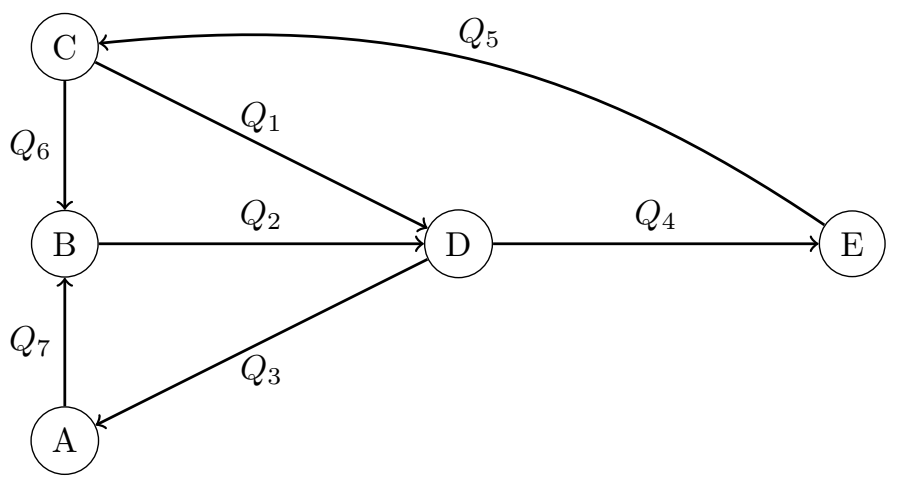

Figure 1: An example of system

Flow rates $\left(Q_{1}, \cdots, Q_{7}\right)$ are functions of space $x \in[0,1]$ and time $t \geq 0$ and are all subject (1.4). To hope for a well-posed problem, it is necessary to define $\left(Q_{1}(t, 0), \cdots, Q_{7}(t, 0)\right)$. In this paper, it is given by transfer operators associated to nodes $A, B, C, D, E$. For example, at node $B$, it is physically relevant to impose that the flow rate in channel 2 is the sum of flow rates from channels 6 and 7 :

$$
Q_{2}(t, 0)=Q_{6}(t, 1)+Q_{7}(t, 1):=g_{B}\left(Q_{6}(t, 1), Q_{7}(t, 1)\right) .
$$

For completeness, paragraph [3, Section 4.2] presents another example of such scalar models coupled by the boundary.

More generally, the family of systems we study constitutes a simplified model for more realistic systems. In [3, Chapter 1], typical examples of hyperbolic PDEs with feedback boundary conditions are cited; the telegrapher equations for electrical lines, the shallow water (Saint-Venant) equations for open channels [21], the isothermal Euler equations for gas flow in pipelines or even the Aw-Rascle equations [2] for road traffic. It should be noted that in previous examples, there is often an in-domain coupling which is not present in our model. Moreover, fluxes are not scalar which render the analysis far more complicated. In fact, this paper focuses on a simplified version of those systems in order to introduce techniques helping in the complex study of general systems of conservation and balance laws from [3, Chapter 1].

Additionally, the stabilization of similar systems with non-local terms receive more and more attention. We can cite [5] where the authors add a nonlocal zeroth order term to be stabilized. In this article, uncertainties on parameters and on the state of the system are allowed and an adaptive command built from an observer is designed. In [11], authors propose a spectral analysis to stabilize a scalar linear transport equation with a non-local velocity. The control is localized at the boundary. Then, by a Lyapunov analysis they prove a local stability result for the nonlinear version of the system.

1.2. Linear feedback. For the case where $g=H \in M_{d}(\mathbb{R})$ is a linear operator, the literature is quite rich. 
1.2.1. When the flux is linear. It can be written as $f(R)=\Lambda$

$=\operatorname{diag}\left(\lambda_{1}, \cdots, \lambda_{d}\right)$ with $\lambda_{1}, \cdots, \lambda_{d}>0$ and the problem of stabilization can be treated for the following classical functional spaces $X$ :

a. Sobolev spaces $W^{m, p}([0,1])$ for $m \in \mathbb{N}$ and $p \in[1,+\infty]$.

b. Spaces $C^{m}([0,1])$ with $(m \in \mathbb{N})$.

c. $B V([0,1])$.

Indeed, in [19, Theorem 3.5 p. 275], the authors prove that 0 is globally exponentially stable in spaces $X$ defined above if and only if there exists $\delta>0$ such that

$$
\left\{z \in \mathbb{C} \mid \operatorname{det}\left(I_{d}-\operatorname{diag}\left(e^{-z / \lambda_{1}}, \cdots, e^{-z / \lambda_{d}}\right) H\right)=0\right\} \subset\{z \in \mathbb{C} \mid \operatorname{Re}(z)<-\delta\} .
$$

However, the criteria (1.5) is not stable with respect to $\Lambda$. Indeed, when we take a $H, \Lambda$ verifying (1.5), it is not guarantied that the same holds for $\tilde{\Lambda}$ with $\tilde{\Lambda}$ diagonal and arbitrarily close to $\Lambda[19$, p. 285].

In the same book, Silkowski [19, Theorem 6.1 p. 286] proves that for all Banach spaces $X$ listed above, 0 is globally exponentially stable and that this stability is robust with respect to $\Lambda$ if and only if

$$
\left.\rho_{0}(H):=\max \left\{\rho\left(\operatorname{diag}\left(e^{i \theta_{1}}, \cdots, e^{i \theta_{n}}\right) H\right) \mid \theta_{i} \in \mathbb{R}\right)\right\}<1
$$

where $\rho$ designates the usual spectral radius.

Condition (1.6) is stronger than (1.5). Some works are also available when there is an additional source term coupling the equations in the domain. One can cite papers $[20,21,13,4,14]$ where Lyapunov methods allow to prove exponential stability for the linearized Saint-Venant system.

1.2.2. When the flux is nonlinear for general hyperbolic systems. For some years, many results came out in the case of nonlinear and non scalar fluxes. Only sufficient conditions of stability are given and most of the time this stability is only proved to be local:

a. For $X=C^{m}([0,1])$ with $m \in \mathbb{N}^{*}$, a sufficient condition $[13,28,25]$ is:

$$
\rho_{\infty}(H):=\inf _{\Delta \in D_{d}^{+}(\mathbb{R})}\left|\Delta H \Delta^{-1}\right|_{\infty}<1
$$

where $|\cdot|_{\infty}$ is the canonical infinity norm of matrices and $D_{d}^{+}(\mathbb{R})$ is the set of diagonal positive matrices.

It should be mentioned that in [13, 28, 25], the stability was proved for $m=1$ but the argument can be adapted for any integer $m>0$.

b. For Sobolev space $W^{m, p}([0,1])$ a sufficient condition for stability writes:

$$
\rho_{p}(H):=\inf _{\Delta \in D_{d}^{+}(\mathbb{R})}\left|\Delta H \Delta^{-1}\right|_{p}<1
$$

where $|\cdot|_{p}$ is the canonical $p$ norm of matrices.

The case $p=2$ was treated in [8] and the general case $p \geq 1$ was treated in [10]. Also, it should be mentioned that in $[8,10]$, the stability was proved for $m=2$ but the argument can be adapted for any integer $m>0$.

c. For $B V([0,1])$, few results are known. To the authors' knowledge only [9] deals with this case. They take a $2 \times 2$ system of conservation laws and give a sufficient condition on $H$ to ensure the local $B V$ stability. 
In this article, we also place ourselves in a $B V$ context and find a sufficient condition on $H$ to ensure a global $B V$ stability. Contrary to [9], non scalar fluxes are discarded here. In this case, solutions are only proved to exist for small initial data. This is why, we rather consider scalar decentralized fluxes (see section 1.3) for which solutions exist for any initial data in $B V$. This hypothesis on the flux is all the more important that when we will study saturated feedback laws, the basin of attraction will be estimated. This would not be possible with solutions defined only for small initial data.

1.3. Saturated control law. Let us introduce a matrix $H \in M_{d}(\mathbb{R})$ potentially unstable in the sense that $\rho_{\infty}(H)>1$ (see (1.7)). Then, it is assumed that there exist matrices $B, K \in M_{d}(\mathbb{R})$ such that $\rho_{\infty}(H+B K)<1$. Finally, the following stabilization problem is considered:

$$
\begin{cases}R_{t}+[f(R)]_{x} & =0 \\ R(t, 0) & =H R(t, 1)+B u(t) \\ R(0, x) & =R_{0}(x) .\end{cases}
$$

If $u(t):=K R(t, 1)$, the control is a linear feedback and as $\rho_{\infty}(H+B K)<1$, the solution to (1.8) converges exponentially fast towards zero.

Now suppose that the control is saturated imposing $u(t):=\sigma(K R(t, 1))$ with $\sigma$ defined as a saturation by component $i e$ there exists a $\sigma_{s}>0$ such that:

$$
\forall i \in \llbracket 1, d \rrbracket, x \in \mathbb{R}, \begin{cases}\sigma_{i}(x)=x & \text { if }|x| \leq \sigma_{s} \\ \sigma_{i}(x)=\operatorname{sign}(x) \sigma_{s} & \text { otherwise. }\end{cases}
$$

From criterion (1.7), the system without saturation is locally stable in $C^{m}([0,1])$ with $m \in \mathbb{N}^{*}$. It is natural to ask ourselves if this property of stability is conserved through the saturation. Apart from this theoretical interest, this problem has gained attention in the last few years because of the increasing need of precision for modeling real actuators. Physical controllers cannot provide infinite energy and sometimes, they saturate rendering classical unsaturated models restrictive. To avoid such situations, engineers choose controllers powerful enough to avoid saturation when the system operates in standard conditions. However, over-dimensioning actuators is not optimal in term of mass and cost of operation for many sophisticated systems as satellites for example. Moreover, in some exceptional configurations, actuators could saturate and lead to very dangerous situations; unpredictable via linear theory.

Very few papers consider the effect of saturation on hyperbolic systems. To our knowledge, only [27] deals with this question in an $H^{1}$ context and for the wave equation. Fortunately, the theory is much more developed for finite dimensional systems where polytopic and deadzone techniques were designed [30].

In this paper, we argue that in a $B V$ context, it is not possible to get a basin of attraction bigger than the region of linearity. We rather prove an $L^{\infty}$ local stability result with an estimation of the basin of attraction. Then, the exponential decay of the $B V$ norm is shown, for solutions whose initial data belongs to the $L^{\infty}$ basin of attraction.

1.4. Scalar conservation laws. The feedback laws being presented, we can now focus on the partial differential equation in itself. The flux $f$ verifies the following Hypothesis 1.1 of regularity:

Hypothesis 1.1. For all $i \in \llbracket 1, d \rrbracket, f_{i} \in C^{1}(\mathbb{R})$ and there exist $\alpha_{i}, \beta_{i}>0$; 


$$
\forall i \in \llbracket 1, d \rrbracket, \alpha_{i} \leq f_{i}^{\prime} \leq \beta_{i}
$$

Such hypothesis allows to define the maximal and the minimal velocity:

$$
\begin{cases}c_{\max } & :=\max _{i \in \llbracket 1, d \rrbracket} \beta_{i} \\ c_{\min } & :=\min _{i \in \llbracket 1, d \rrbracket} \alpha_{i} .\end{cases}
$$

The aim of this section is to give a very short introduction to scalar conservation laws without giving any proof (see [6] for more details).

1.4.1. The set of functions with bounded variations. It is well-known that the space $B V$ is well-adapted for conservation laws (see [6] for instance). This is why, we give the definition and main properties of such a space here:

Definition 1.2. Let $R:[0,1] \mapsto \mathbb{R}^{d}$ be a vector valued function. We say that $R$ has bounded variations if

$$
\forall n \in \mathbb{N}, \forall x_{1}<\ldots<x_{n} \in[0,1], \sum_{i=1}^{n-1}\left|R\left(x_{i+1}\right)-R\left(x_{i}\right)\right|<\infty
$$

where $|\cdot|$ is the canonical euclidean norm.

$$
\text { We denote } T V_{[0,1]}(R)=\sup _{n,\left(x_{1}, \ldots, x_{n}\right)}\left\{\sum_{i=1}^{n-1}\left|R\left(x_{i+1}\right)-R\left(x_{i}\right)\right|\right\} \text { the total variation }
$$

of $R . B V([0,1])$ is the space of vector valued functions with bounded variations and it is a Banach space when $B V([0,1])$ is embedded with the norm $\|\cdot\|_{B V([0,1])}$ defined as

$$
\forall R \in B V([0,1]),\|R\|_{B V([0,1])}=T V_{[0,1]}(R)+\|R\|_{L^{1}([0,1])} .
$$

The reason why we consider this space is because any function with bounded variations has a left and a right limit at each point $x$ of $[0,1]$. Hence, it is easy to define the trace operator and impose a boundary condition. Moreover, $B V([0,1])$ has a very interesting property of compactness which will be very useful when we will pass to the limit in the Lyapunov analysis of approximating solutions. These properties are summed up in a lemma and a theorem:

Lemma 1.3. Let $R:[0,1] \mapsto \mathbb{R}^{d}$ with bounded variations. Then for all $x \in(0,1)$, the left and right limit

$$
R\left(x^{-}\right)=\lim _{y \rightarrow x^{-}} R(y), \quad R\left(x^{+}\right)=\lim _{y \rightarrow x^{+}} R(y)
$$

exist.

Moreover, $R\left(0^{+}\right)$and $R\left(1^{-}\right)$are also well defined and $R$ has at most countably many point of discontinuities.

Proof. This is an adaptation of [6, Lemma 2.1].

Defining the value of $R$ at each jump by $R(x)=R\left(x^{+}\right)$, we can say that $R$ is right continuous in the $L^{1}$ equivalence class. The following theorem is from Helly and states the compactness of $B V([0,1])$ in $L_{l o c}^{1}\left(\mathbb{R}^{+}, L^{1}([0,1])\right)$. 
THEOREM 1.4. [6, Theorem 2.4] Let $\left(R_{\nu}\right)_{\nu}$ be a sequence of functions from $\mathbb{R}^{+} \times$ $[0,1]$ into $\mathbb{R}^{d}$ such that there exist constants $C, M$ and $L$ satisfying

$$
\forall \nu>1, \forall x \in[0,1], \forall t \geq 0, T V_{[0,1]}\left(R_{\nu}(t, .)\right) \leq C,\left|R_{\nu}(t, x)\right| \leq M
$$

and

$$
\forall 0 \leq t, s \leq T,\left\|R_{\nu}(t, .)-R_{\nu}(s, .)\right\|_{L^{1}([0,1])} \leq L|t-s|
$$

Then there exists a subsequence $\left(R_{\mu}\right)_{\mu}$ converging strongly toward a certain $R$ in $L_{\text {loc }}^{1}\left(\mathbb{R}^{+}, L^{1}([0,1])\right)$ and this limit satisfies (1.11)-(1.12) with $R_{\nu}$ replaced by $R$.

1.4.2. Entropy. The concept of entropy is primordial in order to guaranty uniqueness of solutions to conservation laws. This is why we recall some basic definitions in this section.

If one considers the conservation law $R_{t}+[f(R)]_{x}=0$ in the usual weak sense:

$$
\forall \phi \in C_{c}^{1}\left((0, T) \times(0,1) ; \mathbb{R}^{d}\right), \int_{0}^{T} \int_{0}^{1}\left(\phi_{t} R+\phi_{x} f(R)\right)=0,
$$

it is commonly known that this PDE (associated with fixed boundary and initial conditions) can have several weak solutions (see Example 4.3 from [6]). In order to restrain the set of solutions, an entropy functional was introduced ( [12], [23]) and is defined as follows:

Definition 1.5. A continuously differentiable convex function $\eta: \mathbb{R}^{d} \mapsto \mathbb{R}$ is called an entropy for the conservation law $R_{t}+[f(R)]_{x}=0$ with entropy flux $q$ : $\mathbb{R}^{d} \mapsto \mathbb{R}$, if

$$
\forall R \in \mathbb{R}^{d}, D \eta(R) \cdot D f(R)=D q(R) .
$$

For scalar conservation laws of the form $u_{t}+\left[f_{1}(u)\right]_{x}=0$, every convex function is an entropy and the usual choice is $\eta(u):=|u-k|$ with flux $q(u):=\left(f_{1}(u)-\right.$ $\left.f_{1}(k)\right) \operatorname{sign}(u-k)$ where $k$ is an arbitrary real. Knowing this, we introduce the notion of entropy solution to (1.1).

Definition 1.6. Under Hypothesis 1.1, we say that $R \in L_{l o c}^{\infty}\left(\mathbb{R}^{+}, B V([0,1])\right)$ is an entropy solution on $[0, T]$ to the system

$$
\left\{\begin{aligned}
R_{t}+[f(R)]_{x} & =0 \\
R(., 0) & =g(R(., 1)) \\
R(0, .) & =R_{0} \in B V([0,1])
\end{aligned}\right.
$$

if:

$\forall k \in \mathbb{R}^{d}, \sum_{i=1}^{d} \int_{0}^{T} \int_{0}^{1}\left\{\left|R_{i}-k_{i}\right| \phi_{t}+\left(f_{i}\left(R_{i}\right)-f_{i}\left(k_{i}\right)\right) \operatorname{sign}\left(R_{i}-k_{i}\right) \phi_{x}\right\} d x d t \geq 0$

for all $\phi \geq 0$ and $\phi \in C_{c}^{1}((0, T) \times(0,1) ; \mathbb{R})$.

- $R(0,)=.R_{0}$ in the almost everywhere sense.

- $R\left(., 0^{+}\right)=g\left(R\left(., 1^{-}\right)\right)$in the almost everywhere sense. 
Remark 1.7. Here the entropy functional and its flux are defined for all $k$ in $\mathbb{R}^{d}$ by

$$
\forall R \in \mathbb{R}^{d}, \eta_{k}(R)=\sum_{i=1}^{d}\left|R_{i}-k_{i}\right|, q_{k}(R)=\sum_{i=1}^{d}\left(f_{i}\left(R_{i}\right)-f_{i}\left(k_{i}\right)\right) \operatorname{sign}\left(R_{i}-k_{i}\right) .
$$

Moreover, equation (1.14) can be rewritten as

$$
\eta_{k}(R)_{t}+q_{k}(R)_{x} \leq 0
$$

in a weak sense. Hence entropy solutions are the solutions of (1.1) which make the entropy $\eta$ decrease.

1.5. The contribution. Now that all the notions have been introduced, we can be more specific concerning the main contributions of this paper:

- State and prove a well-posedness result of (1.1) in a $B V$ context.

To help us in the task, we use front tracking techniques from DiPerna [22] and Bressan [6] to get an entropy solution in the domain considered. To deal with the boundary condition, the article [9] is the reference work. One could use results from [9] for which well-posedness is proven for system of $2 \times 2$ equations. Here, the proof is simpler and adapted to the context of scalar equations.

- State and prove a global exponential stability result for linear feedback laws. To our knowledge, no global stabilization result holds for feedback laws of the form $R(t, 0)=H R(t, 1)$ in a $B V$ entropy context. The article [26] proposes also a feedback law of the form $R(t, 0)=g\left(\|R(t, .)\|_{L^{1}}\right)$. However, in physical systems the $L^{1}$ norm of the solution is not always accessible by observations. Additionally, the article [9] which considers a $2 \times 2$ system of conservation laws gives only a local stabilization result for an entropy solution.

- The key result of this paper is the statement and the proof of a local exponential stability result for saturated feedback laws. We will see that this is not possible in a $B V$ context. To our knowledge, only [17] has studied this kind of saturated feedback laws in an $L^{\infty}$ context and for the case of constant characteristic velocities.

1.6. Outline. In Section 2, we present and prove an approximation and a wellposedness result for the entropy $B V$ solution to (1.1). The technique of front tracking are mainly used. Then in Section 3, a sufficient condition for global $B V$ stability is given in the case of a linear feedback. Additionally, we give a sufficient condition for the local $L^{\infty}$ stability in the case of a saturated feedback with an estimation of the basin of attraction. Finally, Section 4 is devoted to concluding remarks and perspectives.

Notation: For all $R \in \mathbb{R}^{d},|R|$ designates the canonical euclidean norm of $R$. For matrices $M \in M_{d}(\mathbb{R}),|M|=\sup _{|R|=1, R \in \mathbb{R}^{d}}|M R|$ For all matrices $M \in M_{d}(\mathbb{R}),|M|_{\infty}:=$ $\max _{i=1 . . d} \sum_{j=1}^{d}\left|M_{i, j}\right| . \quad D_{d}^{+}(\mathbb{R})$ is the set of diagonal strictly positive matrices. The value $\rho_{\infty}(M)$ for matrices $M \in M_{d}(\mathbb{R})$ is defined by

$$
\rho_{\infty}(M):=\inf _{\Delta \in D_{d}^{+}(\mathbb{R})}\left|\Delta M \Delta^{-1}\right|_{\infty} . L^{p} \text { spaces on }[0,1](1 \leq p \leq \infty, p \in \mathbb{N})
$$
are embedded with their canonical norms $\|\cdot\|_{L^{p}}$. For all matrices $P \in D_{d}^{+}(\mathbb{R})$ and $R \in L^{\infty}([0,1]),\|R\|_{\infty, P}:=\|P R\|_{L^{\infty}}$. The function $E: \mathbb{R} \mapsto \mathbb{N}$ is the integer part function and the function sign is the usual sign function with $\operatorname{sign}(0)=0$. 
2. Well-posedness and approximation results. This section is devoted to the well-posedness of (1.13). Additionally, we prove the existence of a suitable approximation by piecewise constant functions of the solution to (1.13). This sequence of approximation is crucial for the stability analysis.

2.1. Piecewise constant entropy solutions. Piecewise constant functions play an important role in the theory of BV solutions to conservation laws. Let us recall the definition of what a piecewise constant function is in our context.

Definition 2.1. An element $R$ of $L_{\text {loc }}^{\infty}\left(\mathbb{R}^{+}, B V([0,1])\right)$ is piecewise constant if for all $T>0, R$ viewed as a function defined on $[0, T] \times[0,1]$ is constant on a finite number of polyhedra. The edges of such polyhedra are called the fronts of $R$. Additionally, the absolute value of the jump across the front is called the intensity of the front.

In this paper, the concept of approximating sequence of piecewise constant functions $(\mathrm{PCF})$ is used in the proof of stability and well-posedness.

DEFINITION 2.2. $\left(R_{\nu}\right)_{\nu}$ is an approximating sequence of PCFs of an entropy solution $R$ to (1.13) if:

- For $\nu>1$ fixed, $R_{\nu}$ is piecewise constant in the sense of Definition 2.1 and takes its values in $2^{-(n+1) \nu} \mathbb{Z}$ on strips

$$
\left\{(x, t) \mid 0 \leq x \leq 1, \max \left\{(x+n-1) / c_{\max }, 0\right\} \leq t \leq(x+n) / c_{\max }\right\}
$$

for $n \in \mathbb{N}$. The velocities of fronts are all bounded from below by $c_{\min }$ and from above by $c_{\max }$ (see (1.9) for the definition of $c_{\min }$ and $c_{\max }$ ).

- For $\nu>1$ fixed, no more than one front at a time can interact with the right boundary.

- For $\nu>1$ fixed, if at a time $t \geq 0$ several fronts interact, the sum of intensities of outgoing fronts is inferior to the sum of intensities of ingoing fronts.

- The sequence $\left(R_{\nu}(0, .)\right)_{\nu}$ converges toward $R_{0}$ in $B V([0,1])$.

- The approximated boundary condition is verified:

$$
\forall n \in \mathbb{N}, \forall t \text { s.t } \frac{n}{c_{\max }} \leq t \leq \frac{n+1}{c_{\max }}, R_{\nu}\left(t, 0^{+}\right)=g_{(n+2) \nu}\left(R_{\nu}\left(t, 1^{-}\right)\right)
$$

where:

$$
\forall R \in \mathbb{R}^{d}, \forall \nu>1, g_{\nu}(R)=2^{-\nu}\left(E\left(2^{\nu} g(R)\right)\right) .
$$

- $\forall t \geq 0, \Delta t>0$,

$$
T V_{[0,1]}(R(t, .)) \leq \limsup _{\nu \rightarrow+\infty} \sup _{s \in[t, t+\Delta t]} T V_{[0,1]}\left(R_{\nu}(s, .)\right)
$$

and

$$
\|R(t, .)\|_{L^{\infty}([0,1])} \leq \limsup _{\nu \rightarrow+\infty} \sup _{s \in[t, t+\Delta t]}\left\|R_{\nu}(s, .)\right\|_{L^{\infty}([0,1])}
$$


2.2. The result of well-posedness and approximation. Now we give the first result of this paper:

TheOREM 2.3. Under Hypothesis 1.1 and for all $R_{0} \in B V([0,1]), g \in \operatorname{Lip}\left(\mathbb{R}^{d}, \mathbb{R}^{d}\right)$, there exists a unique entropy solution $R \in L_{l o c}^{\infty}\left(\mathbb{R}^{+}, B V([0,1])\right)$ to (1.13). Moreover, there exists an approximating sequence of $P C F\left(R_{\nu}\right)_{\nu}$ of the entropy solution $R$.

Proof. A proof is given in Appendix A for the existence and Appendix B for the uniqueness.

3. Lyapunov analysis. Before going into the stability analysis, the functional $T V_{H}$ defined on the space $\mathrm{BV}$, is intoduced. For all matrices $H$ in $M_{d}(\mathbb{R})$, it is defined as follows:

$$
\forall R \in B V([0,1]), T V_{H}(R)=T V_{[0,1]}(R)+\left|H R\left(1^{-}\right)-R\left(0^{+}\right)\right|,
$$

where $R\left(1^{-}\right)$and $R\left(0^{+}\right)$has to be understood as the left and right limits of the function $R$ at $x=1$ and $x=0$.

Moreover, the Hypothesis 3.1 is imposed:

Hypothesis 3.1. The feedback matrix $H$ verifies:

$$
\rho_{\infty}(H)<1 \text {. }
$$

Remark 3.2. By [9, Remark 1.4],

$$
\forall M \in M_{d}(\mathbb{R}), \rho_{\infty}(M)=\rho_{1}\left(M^{T}\right)=\rho_{1}(M)=\rho_{\infty}\left(M^{T}\right) .
$$

The following lemma ensures the equivalence between $T V_{H}$ and $\|\cdot\|_{B V([0,1])}$.

LEMma 3.3. Assume Hypothesis 3.1. The functional $T V_{H}$ defined in (3.1) is a norm on $B V([0,1])$ equivalent to the norm $\|\cdot\|_{B V([0,1])}$ defined in (1.10). Moreover, there exists a constant $C>0$ such that

$$
\forall R \in B V([0,1]),\|R\|_{L^{\infty}([0,1])} \leq C T V_{H}(R) .
$$

Proof. We first prove the following claim:

$$
\forall R \in \mathbb{R}^{d},|R| \leq C|R-H R| .
$$

Let $P \in D_{d}^{+}(\mathbb{R})$ such that

$$
\left|P H P^{-1}\right|_{\infty}<1
$$

The map $\|\cdot\|_{\infty}:\left\{\begin{array}{lll}M_{d}(\mathbb{R}) & \rightarrow & \mathbb{R}^{+} \\ M & \mapsto & \left|P M P^{-1}\right|_{\infty}\end{array} \quad\right.$ defines an algebra norm on $M_{d}(\mathbb{R})$ and $\|H\|_{\infty}<1$. Hence, $I_{d}-H$ is invertible, which gives (3.3) with $C:=\left|(I-H)^{-1}\right|$.

$$
\begin{aligned}
T V_{H}(R) & =T V_{[0,1]}(R)+\left|H R\left(1^{-}\right)-R\left(0^{+}\right)\right| \\
& \leq T V_{[0,1]}(R)+\left|H R\left(1^{-}\right)-H R\left(0^{+}\right)\right|+\left|H R\left(0^{+}\right)-R\left(0^{+}\right)\right| \\
& \leq T V_{[0,1]}(R)+\left|H \| R\left(1^{-}\right)-R\left(0^{+}\right)\right|+\left|H-I_{d}\right|\left|R\left(0^{+}\right)\right| \\
& \leq(1+|H|) T V_{[0,1]}(R)+\left|H-I_{d}\right|\left|R\left(0^{+}\right)\right| .
\end{aligned}
$$


Take $x \in[0,1]$, by the triangle inequality,

$$
\begin{aligned}
T V_{H}(R) & \leq(1+|H|) T V_{[0,1]}(R)+\left|H-I_{d}\right|\left|R\left(0^{+}\right)-R(x)\right|+\left|H-I_{d}\right||R(x)| \\
& \leq\left(1+|H|+\left|H-I_{d}\right|\right) T V_{[0,1]}(R)+\left|H-I_{d}\right||R(x)| .
\end{aligned}
$$

Integrating with respect to $x$ on $[0,1]$, one obtains:

$$
\begin{aligned}
T V_{H}(R) & \leq\left(1+|H|+\left|H-I_{d}\right|\right) T V(R)+\left|H-I_{d}\right|\|R\|_{L^{1}([0,1])} \\
& =C\|R\|_{B V([0,1])} .
\end{aligned}
$$

where $C=1+|H|+\left|H-I_{d}\right|$.

To get the converse inequality, we remark that by (3.3),

$$
\left|R\left(1^{-}\right)\right| \leq C\left|H R\left(1^{-}\right)-R\left(1^{-}\right)\right| .
$$

As a consequence,

$$
\begin{aligned}
\|R\|_{B V([0,1])} & =T V_{[0,1]}(R)+\|R\|_{L^{1}([0,1])} \\
& \leq T V_{[0,1]}(R)+\left|R\left(1^{-}\right)\right|+\left\|R-R\left(1^{-}\right)\right\|_{L^{1}([0,1])} \\
& \leq 2 T V_{[0,1]}(R)+C\left|H R\left(1^{-}\right)-R\left(1^{-}\right)\right| \\
& \leq 2 T V_{[0,1]}(R)+C\left|H R\left(1^{-}\right)-R\left(0^{+}\right)\right|+C\left|R\left(0^{+}\right)-R\left(1^{-}\right)\right| \\
& \leq(2+C) T V_{[0,1]}(R)+C\left|H R\left(1^{-}\right)-R\left(0^{+}\right)\right|
\end{aligned}
$$

and both norms are equivalent. Concerning the $L^{\infty}$ estimate (3.2), take a couple $(x, y) \in[0,1]^{2}$ and using again the triangle inequality

$$
|R(x)| \leq|R(x)-R(y)|+|R(y)| \leq T V_{[0,1]}(R)+|R(y)| .
$$

Integrating with respect to $y$ on $[0,1]$, one gets

$$
|R(x)| \leq T V_{[0,1]}(R)+\|R\|_{L^{1}([0,1])}=\|R\|_{B V([0,1])} .
$$

And as this is true for all $x$ in $[0,1]$,

$$
\|R\|_{L^{\infty}([0,1])} \leq\|R\|_{B V([0,1])} .
$$

The equivalence between the norms $\|\cdot\|_{B V([0,1])}$ and $T V_{H}$ proved earlier allows to deduce (3.2).

3.1. Lyapunov analysis for the unsaturated system. In this section, we consider the following system

$$
\begin{cases}R_{t}+[f(R)]_{x} & =0 \\ R(., 0) & =H R(., 1) \\ R(0, .) & =R_{0} \in B V([0,1])\end{cases}
$$

where the feedback operator $g$ presented in the introduction is replaced by a matrix $H \in M_{d}(\mathbb{R})$.

The main theorem of this section is presented here: 
TheOREm 3.4. Under Hypothesis 3.1 and if $0<\gamma<-\log \left(\rho_{\infty}(H)\right)$, then the unique entropy solution of (3.4) satisfies

$$
\forall t \geq 0,\|R\|_{B V([0,1])} \leq C e^{-\gamma c_{\min } t}\left\|R_{0}\right\|_{B V([0,1])}
$$

where $C>0$ is a constant which does not depend on $R_{0}$ and $t$.

A candidate Lyapunov functional first introduced by Glimm [18] and then by Coron et al [9] applies well to piecewise constant functions and is defined by:

DeFINITION 3.5. Let $R$ be a piecewise constant function on $[0,1]$ and taking its values in $\mathbb{R}^{d}$. Take $i \in \llbracket 1, d \rrbracket$ :

- We denote $x_{i, 1}<x_{i, 2}<\cdots<x_{i, n_{i}}$ the discontinuities of $R_{i}$ ( $n_{i}$ being the number of discontinuities).

- For all $j \in \llbracket 1, n_{i} \rrbracket, r_{i, j}^{l}, r_{i, j}^{r}$ designate the respective left and right state of $R_{i}$ around $x_{i, j}$.

The Lyapunov functional $\mathcal{L}$ evaluated at $R$ writes

$$
\mathcal{L}(R)=\sum_{i=1}^{d} P_{i} \sum_{j=1}^{n_{i}}\left|r_{i, j}^{r}-r_{i, j}^{l}\right| e^{-\gamma x_{i, j}}+\sum_{i=1}^{d} P_{i}\left|[H R]_{i}\left(1^{-}\right)-R_{i}\left(0^{+}\right)\right|
$$

where $\gamma>0$ and $P=\operatorname{diag}\left\{P_{i}, i \in \llbracket 1, d \rrbracket\right\} \in D_{d}^{+}(\mathbb{R})$ will be selected later.

Remark 3.6. Obviously, there exists a constant $C(H, P, \gamma)>1$ such that for all $R$ piecewise constant:

$$
\frac{\mathcal{L}(R)}{C(H, P, \gamma)} \leq T V_{H}(R) \leq C(H, P, \gamma) \mathcal{L}(R)
$$

Remark 3.7. In our case, the boundary terms in (3.5) are not zero since the boundary condition is approximated by (2.1).

Theorem 3.4 is proved using a piecewise approximation of the solution for which the exponential decay of the Lyapunov functional $\mathcal{L}$ is established. As a last step, we pass to the limit.

Proof. We consider $\left(R_{\nu}\right)_{\nu}$ an approximating sequence of PCFs of the entropy solution $R$ in the sense of Definition 2.2. Such a sequence exists by Theorem 2.3. The following lemma asserts the exponential stability of the approximation:

Lemma 3.8. If $0<\gamma<-\log \left(\rho_{\infty}(H)\right)$. Then, for all $P \in D_{d}^{+}\left(\mathbb{R}^{d}\right)$ such that $\left|P^{-1} H^{T} P\right|_{\infty}<e^{-\gamma}$, there exists $\tilde{\nu}(P, H, \gamma)$ such that

$$
\forall \nu>\tilde{\nu}, \forall t \geq 0, \mathcal{L}\left(R_{\nu}\right) \leq e^{-\gamma c_{\min } t} \mathcal{L}\left(R_{0, \nu}\right)+\frac{E\left(c_{\max } t\right)+1}{2^{\nu}} \sum_{i=1}^{d} P_{i}
$$

Proof. Fix $\nu>1, P \in D_{d}^{+}\left(\mathbb{R}^{d}\right)$ such that $\left|P^{-1} H^{T} P\right|_{\infty}<e^{-\gamma}$ and time $0 \leq t \leq$ $1 / c_{\max }$.

Three cases are to be considered: 
- (Case 1) If at time $t$ there is no interaction between two fronts nor between a front and the boundary, then $\mathcal{L}\left(R_{\nu}\right)$ is differentiable and because the boundary term is constant locally around $t$ one gets:

$$
\begin{aligned}
\frac{d \mathcal{L}\left(R_{\nu}(t, .)\right)}{d t} & =-\gamma \sum_{i=1}^{d} P_{i} \sum_{j=1}^{n_{i}} \frac{d x_{i, j}}{d t}\left|r_{i, j}^{r}-r_{i, j}^{l}\right| e^{-\gamma x_{i, j}} \\
& \leq-\gamma c_{\min } \sum_{i=1}^{d} P_{i} \sum_{j=1}^{n_{i}}\left|r_{i, j}^{r}-r_{i, j}^{l}\right| e^{-\gamma x_{i, j}}
\end{aligned}
$$

Here, we used the fact that for all integers $i \in \llbracket 1, d \rrbracket$, characteristic velocities $\frac{d x_{i, j}}{d t}$ are bounded from below by $c_{\min }>0$. Finally, by the definition of $\left.\mathcal{L}\left(R_{\nu}(t,).\right)\right)$

$$
\begin{aligned}
\frac{d \mathcal{L}\left(R_{\nu}(t, .)\right)}{d t} \leq & \left.-\gamma c_{\min } \mathcal{L}\left(R_{\nu}(t, .)\right)\right) \\
& +\gamma c_{\min } \sum_{i=1}^{d} P_{i}\left|\left[H R_{\nu}\right]_{i}\left(t, 1^{-}\right)-R_{\nu, i}\left(t, 0^{+}\right)\right| \\
\leq & \left.-\gamma c_{\min } \mathcal{L}\left(R_{\nu}(t, .)\right)\right)+\frac{\gamma c_{\min }}{2^{\nu}} \sum_{i=1}^{d} P_{i}
\end{aligned}
$$

where we used (2.1) with $g$ replaced by $H$ to get last equation.

- (Case 2) When a front interaction happens, the total variation is non increasing by construction and as a consequence

$$
\mathcal{L}\left(R_{\nu}\left(t^{+}, .\right)\right)-\mathcal{L}\left(R_{\nu}\left(t^{-}, .\right)\right) \leq 0 .
$$

Here we used the third point of Definition 2.2.

- (Case 3) When an interaction of a front with the boundary happens, computations are a bit more difficult. Suppose that such a front is of type $i \in \llbracket 1, d \rrbracket$ and has $\left(R_{i, l}, R_{i, r}\right)$ as respective left and right state (see Figure 2). We note its intensity by $I_{i}:=\left|R_{i, l}-R_{i, r}\right|$. Note that as $R_{\nu}$ takes its values in $2^{-\nu} \mathbb{Z}$ on the triangle $\left\{(x, t) \mid 0<t<x / c_{\max }\right\}$ :

$$
I_{i} \geq 2^{-\nu} .
$$

Moreover, recall that simultaneous interactions of fronts with the boundary are forbidden by construction. Using the approximate boundary condition (2.1) with $g$ replaced by the linear operator $H$, it holds

$$
\begin{aligned}
\mathcal{L}\left(R_{\nu}\left(t^{+}, .\right)\right)-\mathcal{L}\left(R_{\nu}\left(t^{-}, .\right)\right) \leq & \sum_{j=1}^{d} P_{j}\left|H_{j, i}\left(R_{i, r}-R_{i, l}\right)\right|-e^{-\gamma} I_{i} P_{i} \\
& +2^{-2 \nu+2} \sum_{j=1}^{d} P_{j} .
\end{aligned}
$$

The second term on the right-hand side of (3.10) corresponds to the leaving front (which is of type $i$ ). The first term results from the entering fronts at the left boundary. Note that an entering front of type $j \in \llbracket 1, d \rrbracket$ may rather be a fan of fronts (see Figure 2). This is not problematic because the sum of the intensities of the fronts composing the fan is equal to the difference 
of extremal states of the fan by construction (see Appendix A for details). The last term in (3.10) corresponds to the approximation of the boundary condition (2.1).

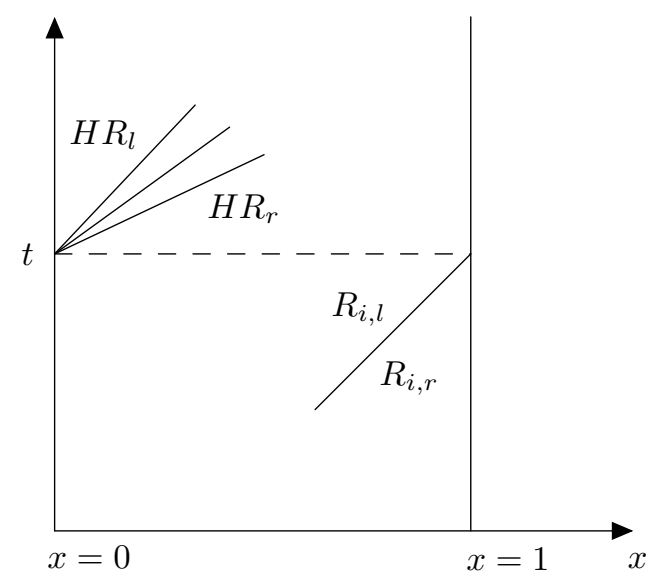

Figure 2: Case 3

Then, using the definition of $|\cdot|_{\infty}$ and (3.9), one gets:

$$
\begin{aligned}
\mathcal{L}\left(R_{\nu}\left(t^{+}, .\right)\right)-\mathcal{L}\left(R_{\nu}\left(t^{-}, .\right)\right) \leq & \left(\sum_{j=1}^{d} \frac{P_{j}}{P_{i}}\left|H_{j, i}\right|-e^{-\gamma}\right) P_{i} I_{i} \\
& +2^{-\nu+2} \sum_{j=1}^{d} P_{j} I_{i} \\
\leq & \left(\left|P^{-1} H^{T} P\right|_{\infty}+2^{-\nu+2} \sum_{j=1}^{d} P_{j} / P_{i}\right. \\
& \left.-e^{-\gamma}\right) P_{i} I_{i} .
\end{aligned}
$$

Remark 3.9. Here we see why the approximated boundary condition (2.1) is essential. Thanks to it, the error term $2^{-2 \nu+2} \sum_{j=1}^{d} P_{j}$ coming from the approximation of $g$ by $g_{\nu}$ can be bounded by the intensity $I_{i} \geq 2^{-\nu}$ of the front hitting the right boundary.

As $\left|P^{-1} H^{T} P\right|_{\infty}-e^{-\gamma}<0$ by assumption, we can take $\nu$ sufficiently large say $\nu \geq \tilde{\nu}(P, H, \gamma)$ such that

$$
\mathcal{L}\left(R_{\nu}\left(t^{+}, .\right)\right)-\mathcal{L}\left(R_{\nu}\left(t^{-}, .\right)\right) \leq 0
$$

(Case 2) and (Case 3) can occur only a finite number of times on finite time intervals because $R_{\nu}$ is piecewise constant in the sense of Definition 2.1. Consequently, one can integrate (3.8) with respect to time to get:

$$
\forall 0 \leq t \leq 1 / c_{\max }, \mathcal{L}\left(R_{\nu}(t, .)\right) \leq e^{-\gamma c_{\min } t} \mathcal{L}\left(R_{0, \nu}\right)+\frac{1}{2^{\nu}} \sum_{i=1}^{d} P_{i} .
$$

For time $n / c_{\max } \leq t \leq(n+1) / c_{\max }$ where $n$ is an integer, one easily proves by induction that: 


$$
\forall n / c_{\max } \leq t \leq(n+1) / c_{\max } \mathcal{L}\left(R_{\nu}(t, .)\right) \leq e^{-\gamma c_{\min } t} \mathcal{L}\left(R_{0, \nu}\right)+\frac{n+1}{2^{\nu}} \sum_{i=1}^{d} P_{i} .
$$

This ends the proof of Lemma 3.8.

Now, we conclude on the proof of Theorem 3.4 taking $t \geq 0$ fixed. By (3.7) and (3.6), there exists a constant $C>0$ such that

$$
\forall \nu>0, T V_{H}\left(R_{\nu}(t, .)\right) \leq C\left(e^{-\gamma c_{\min } t} T V_{H}\left(R_{0, \nu}\right)+\frac{E\left(c_{\max } t\right)+1}{2^{\nu}} \sum_{i=1}^{d} P_{i}\right) .
$$

Using the equivalence between the norm $T V_{H}$ and the norm $\|\cdot\|_{B V([0,1])}$,

$$
\forall \nu>0,\left\|R_{\nu}(t, .)\right\|_{B V([0,1])} \leq C\left(e^{-\gamma c_{\min } t}\left\|R_{0, \nu}\right\|_{B V([0,1])}+\frac{E\left(c_{\max } t\right)+1}{2^{\nu}} \sum_{i=1}^{d} P_{i}\right)
$$

where the constant $C>0$ may have changed.

As $\left(R_{\nu}\right)_{\nu}$ is an approximating sequence of PCFs of $R$, one has:

$$
\left\{\begin{array}{cl}
\lim _{\nu \rightarrow \infty} R_{\nu}(0, .) & =R_{0} \in B V([0,1]) \\
\forall \tau \geq 0, d \tau>0, T V_{[0,1]}(R(\tau, .)) & \leq \limsup _{\nu \rightarrow \infty} \sup _{s \in[\tau, \tau+d \tau]} T V_{[0,1]}\left(R_{\nu}(s, .)\right) .
\end{array}\right.
$$

Moreover, by Remark A.4,

$$
\forall \tau \geq 0, \lim _{\nu \rightarrow \infty}\left\|R_{\nu}(\tau, .)-R(\tau, .)\right\|_{L^{1}([0,1])}=0 .
$$

We have for all $d t>0$,

$$
\begin{aligned}
\|R(t, .)\|_{B V([0,1])} & \leq \limsup _{\nu \rightarrow \infty}\left(\sup _{s \in[t, t+d t]} T V_{[0,1]}\left(R_{\nu}(s, .)\right)+\left\|R_{\nu}(t, .)\right\|_{L^{1}([0,1])}\right) \\
& \leq \limsup _{\nu \rightarrow \infty} \sup _{s \in[t, t+d t]}\left(T V_{[0,1]}\left(R_{\nu}(s, .)\right)+\left\|R_{\nu}(s, .)\right\|_{L^{1}([0,1])}\right) \\
& =\limsup _{\nu \rightarrow \infty} \sup _{s \in[t, t+d t]}\left\|R_{\nu}(s, .)\right\|_{B V([0,1])} \\
& \leq C \limsup _{\nu \rightarrow \infty}\left(e^{-\gamma c_{\min } t}\left\|R_{0, \nu}\right\|_{B V([0,1])}+\frac{E\left(c_{\max } t\right)+1}{2^{\nu}} \sum_{i=1}^{d} P_{i}\right) \\
& =C e^{-\gamma c_{\min } t}\left\|R_{0}\right\|_{B V([0,1])}
\end{aligned}
$$

where (3.11) has been used to get the fourth equation.

This finishes the proof of Theorem 3.4.

3.2. Stability analysis for the saturated system. In this section, we consider the following system:

$$
\begin{cases}R_{t}+[f(R)]_{x} & =0 \\ R(., 0) & =[H \cdot+B \sigma(K \cdot)] R(., 1) \\ R(0, .) & =R_{0} \in B V([0,1])\end{cases}
$$


The deadzone function is defined by:

$$
\forall R \in \mathbb{R}^{d}, \phi(R)=\sigma(R)-R
$$

and Hypothesis 3.10:

Hypothesis 3.10. The matrices $H, B, K$ are chosen such that:

$$
\rho_{\infty}(H+B K)<1
$$

Here the main result is different since we prove local exponential stability (Proposition 3.12). It is not possible to study directly the problem of $B V$ stability because of the lack of contractivity of the saturation $\sigma$.

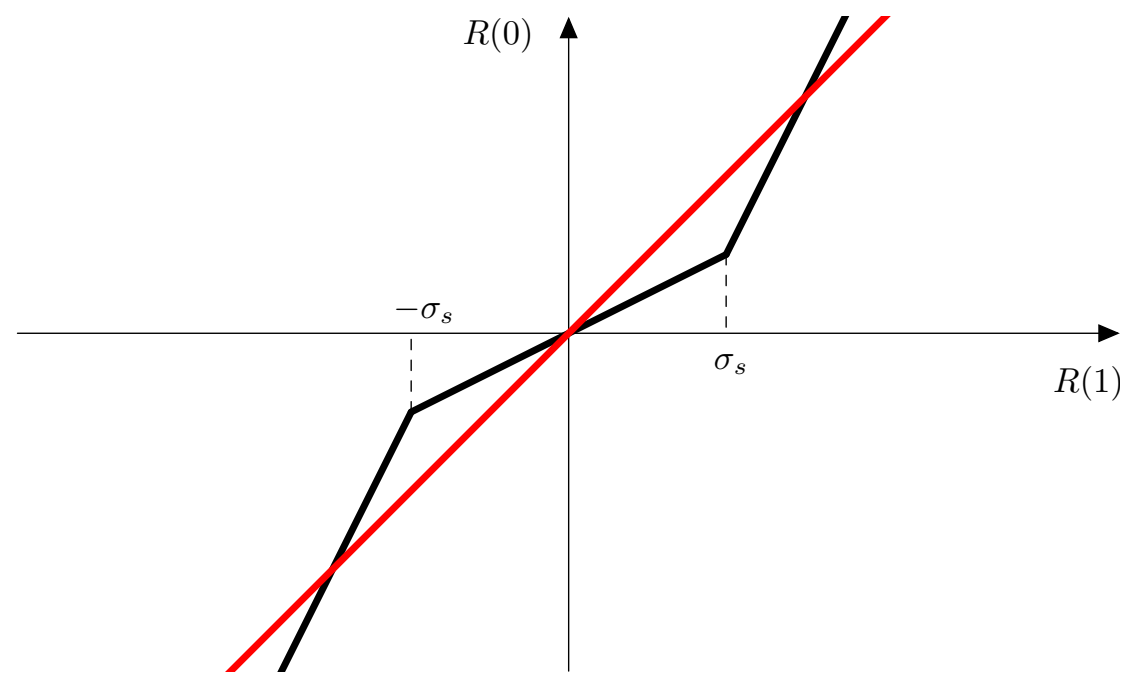

Figure 3: The feedback operator (black line) compared with the graph of the function $R(0)=R(1)$ (red line)

Motivating example 3.11. In Figure 3, we represent the boundary operator $H$. $+B \sigma(K \cdot)$ for $d=1, H=2, B=1, K=-1.5$ and $\sigma_{s}=2$. Except for the zone of linearity, the boundary operator is only 2-Lipschitz. As a consequence, it is possible to construct a front whose left/right states are arbitrary close to the zone of linearity and whose intensity increases after a passage through the feedback operator. This is why it is not possible to get a basin of attraction in $B V$ norm larger than the zone of linearity. We rather prove the $L^{\infty}$ local stability with a basin of attraction in $L^{\infty}$.

This section is devoted to the proof of the following proposition and theorem (the definition of $\|\cdot\|_{\infty, P}$ is given in the section notation):

Proposition 3.12. Under Hypothesis 3.10, if $0<\gamma<-\log \left(\rho_{\infty}(H+B K)\right)$. Then, for all $P \in D_{d}^{+}\left(\mathbb{R}^{d}\right)$ such that $\left|P(H+B K) P^{-1}\right|_{\infty} \leq e^{-\gamma}$, there exists a constant $C$ depending on $(H, B, K, P, \gamma)$ such that if $R_{0} \in B V([0,1])$ and if: 


$$
\left\|R_{0}\right\|_{\infty, P}<\frac{\left|P B P^{-1}\right|_{\infty} P_{\min } \sigma_{s}}{\left.|| P(H+B K) P^{-1}\right|_{\infty}+\left|P B P^{-1}\right|_{\infty}\left|P K P^{-1}\right|_{\infty}-e^{-\gamma} \mid} .
$$

Then, the unique entropy solution $R \in L_{l o c}^{\infty}\left(\mathbb{R}^{+}, B V([0,1])\right)$ of (3.12) satisfies,

$$
\forall t \geq 0,\|R(t, .)\|_{L^{\infty}([0,1])} \leq C e^{-\gamma c_{\min } t}\left\|R_{0}\right\|_{L^{\infty}([0,1])}
$$

where $C$ depends on the parameters of the problem but not $R_{0}$.

For cases where $\rho_{\infty}(H)>1$, the denominator in (3.14) is not zero:

Remark 3.13. If $\rho_{\infty}(H)>e^{-\gamma}$, then we claim that for all $P \in D_{d}^{+}(\mathbb{R})$ :

$$
\left|P(H+B K) P^{-1}\right|_{\infty}+\left|P B P^{-1}\right|_{\infty}\left|P K P^{-1}\right|_{\infty}-e^{-\gamma}>0 .
$$

Proof of the claim of Remark 3.13. Let $P$ be in $D_{d}^{+}(\mathbb{R})$. As $\rho_{\infty}(H)>e^{-\gamma}$,

$$
\left|P H P^{-1}\right|_{\infty}>e^{-\gamma}
$$

This gives by the triangle inequality:

$$
\left|P(H+B K) P^{-1}\right|_{\infty}+\left|P B K P^{-1}\right|_{\infty}>e^{-\gamma} .
$$

Finally, by the fact that

$$
\forall A, B \in M_{d}(\mathbb{R}),\left|P A B P^{-1}\right|_{\infty} \leq\left|P A P^{-1}\right|_{\infty}\left|P B P^{-1}\right|_{\infty},
$$

we have:

$$
\left|P(H+B K) P^{-1}\right|_{\infty}+\left|P B P^{-1}\right|_{\infty}\left|P B P^{-1}\right|_{\infty}>e^{-\gamma}
$$

and the claim is proved.

The following theorem is a consequence of Proposition 3.12 and constitutes a $B V$ exponential stability result.

Theorem 3.14. Under the conditions of Proposition 3.12,

$$
\forall t \geq 0,\|R(t, .)\|_{B V([0,1])} \leq C e^{-\gamma c_{\min } t}\left\|R_{0}\right\|_{B V([0,1])}
$$

where $C$ depends on the parameters of the problem but not $R_{0}$.

Let us assume for the time being Proposition 3.12 and prove Theorem 3.14:

Proof of Theorem 3.14. Equation (3.15) implies that at a certain time denoted $t^{\star}$ depending on $\left\|R_{0}\right\|_{L^{\infty}([0,1])}$, the solution enters in the zone of linearity and stays in it. Then, Theorem 3.4 implies:

$$
\forall t \geq t^{\star},\|R(t, .)\|_{B V([0,1])} \leq C e^{-\gamma c_{\min }\left(t-t^{\star}\right)}\left\|R\left(t^{\star}, .\right)\right\|_{B V([0,1])}
$$

where $C$ depends on $H, B, K, P, \gamma, \sigma_{s}$.

Then, for $t \leq t^{\star}$, one can prove using the same techniques from Section 3.1 that:

$$
\forall 0 \leq t \leq t^{\star},\|R(t, .)\|_{B V([0,1])} \leq e^{\nu t}\left\|R_{0}\right\|_{B V([0,1])}
$$


where $\nu>0$ is a constant depending on $c_{\max }, \gamma$ and a Lipschitz constant of the feedback operator $H+B \sigma(K)$. From (3.17) and (3.16), one gets:

$$
\forall t \geq 0,\|R(t, .)\|_{B V([0,1])} \leq C e^{-\gamma c_{\min } t}\left\|R_{0}\right\|_{B V([0,1])}
$$

where $C$ depends on the parameters of the problem and on $\left\|R_{0}\right\|_{L^{\infty}([0,1])}$. As the bound (3.14) holds, we can conclude that $C$ does not depend on $\left\|R_{0}\right\|_{L^{\infty}([0,1])}$ and the corollary is proved.

The following lemma is useful for the proof of Proposition 3.12.

LEMma 3.15. Let $R \in \mathbb{R}^{d}$ be such that:

$$
|P R|_{\infty} \leq \frac{\left|P B P^{-1}\right|_{\infty} P_{\min } \sigma_{s}}{\left.|| P(H+B K) P^{-1}\right|_{\infty}+\left|P B P^{-1}\right|_{\infty}\left|P K P^{-1}\right|_{\infty}-e^{-\gamma} \mid}
$$

Then,

$$
|P(H R+B \sigma(K R))|_{\infty} \leq e^{-\gamma}|P R|_{\infty} .
$$

Proof. Let $i$ be in $\llbracket 1, d \rrbracket$. If $\operatorname{sat}_{i}(R):=\left\{j \in \llbracket 1, d \rrbracket,||[K R]_{j} \mid>\sigma_{s}\right.$ and $\left.B_{i, j} \neq 0\right\}$ is empty, then:

$$
\begin{aligned}
P_{i}|H R+B \sigma(K R)|_{i} & \left.=P_{i} \mid(H+B K) R\right)\left.\right|_{i} \\
& \leq\left|P(H+B K) P^{-1}\right|_{\infty}|P R|_{\infty} \\
& \leq e^{-\gamma}|P R|_{\infty} .
\end{aligned}
$$

If the set $\operatorname{sat}_{i}(R)$ is not empty, then:

$$
\begin{aligned}
P_{i}|H R+B \sigma(K R)|_{i}= & \left.P_{i} \mid(H+B K) R+B \phi(K R)\right)\left.\right|_{i} \\
\leq & \sum_{j=1}^{d} P_{i}\left|(H+B K)_{i, j} R_{j}\right| \\
& +\sum_{j \in s_{i}(R)}^{d} P_{i}\left|B_{i, j}\right|\left(\left|[K R]_{j}\right|-\sigma_{s}\right) \\
\leq & \sum_{j=1}^{d} P_{i}\left|(H+B K)_{i, j} \frac{1}{P_{j}} P_{j} R_{j}\right| \\
& \left.+\sum_{j \in \operatorname{sat}_{i}(R)}^{d} P_{i}\left|B_{i, j}\right| \frac{P_{j}}{P_{j}}\left|[K R]_{j}\right|-\sigma_{s}\right) \\
\leq & \left|P(H+B K) P^{-1}\right|_{\infty}|P R|_{\infty} \\
& +\left|P B P^{-1}\right|_{\infty}\left(\left|P K P^{-1}\right|_{\infty}|P R|_{\infty}-P_{\min } \sigma_{s}\right) \\
\leq & e^{-\gamma|P R|_{\infty}}
\end{aligned}
$$

where we have used the hypothesis (3.18) to get the last inequality.

Now the focus is on the proof of Proposition 3.12.

Proof of Proposition 3.12. Take $P \in D_{d}^{+}(\mathbb{R})$ such that $\left|P(H+B K) P^{-1}\right|_{\infty}<e^{-\gamma}$ and $R_{0} \in B V([0,1])$ satisfying (3.14). We consider $\left(R_{\nu}\right)_{\nu}$ an approximating sequence of PCFs of the entropy solution $R$ in the sense of Definition 2.2. Such a sequence exists because of Theorem 2.3. Then, we analyze the exponential damping of $R_{\nu}$ for a fixed $\nu>1$. As $\left(R_{0, \nu}\right)_{\nu}$ converges towards $R_{0}$ in $B V([0,1])$, it holds for $\nu$ sufficiently large:

$$
\left\|R_{0, \nu}\right\|_{\infty, P} \leq \frac{\left|P B P^{-1}\right|_{\infty} P_{\min } \sigma_{s}}{\left.|| P(H+B K) P^{-1}\right|_{\infty}+\left|P B P^{-1}\right|_{\infty}\left|P K P^{-1}\right|_{\infty}-e^{-\gamma} \mid}
$$


We first recall the definition of $c_{\min }, c_{\max }$ the respective minimum and maximum velocity, in (1.9). Let $t \leq 1 / c_{\min }$ and $x>c_{\max } t$ be in $[0,1]$. Constructing the light cone enclosed by line with slopes $1 / c_{\min }$ and $1 / c_{\max }$ and passing through $(t, x)$, the following estimate is obtained:

$$
\left|P R_{\nu}(t, x)\right|_{\infty} \leq\left\|R_{0, \nu}\right\|_{\infty, P}
$$

The argument of the light cone can be justified by the fact that the $L^{\infty}$ norm does not increase by fronts interaction (see Appendix Appendix A.2.3) and because fronts velocities belongs to $\left[c_{\min }, c_{\max }\right]$.

When $x \leq c_{\max } t$, constructing the light cone enclosed by lines with slopes $1 / c_{\min }$ and $1 / c_{\max }$ and passing through $(t, x)$, one gets:

$$
\left|P R_{\nu}(t, x)\right|_{\infty} \leq \max \left\{\left\|R_{0, \nu}\right\|_{\infty, P}, \sup _{t \in\left[0,1 / c_{\min }\right]}\left|P R_{\nu}(t, 0)\right|\right\} .
$$

The boundary condition gives:

$$
\left|P R_{\nu}(t, x)\right|_{\infty} \leq \max \left\{\left\|R_{0, \nu}\right\|_{\infty, P}, \sup _{t \in\left[0,1 / c_{\min }\right]}\left|P\left[H \cdot+B \sigma\left(K^{\cdot}\right)\right] R_{\nu}(t, 1)\right|\right\} .
$$

By (3.20) applied to $x=1$ and (3.19), hypothesis of Lemma 3.15 are verified and consequently:

$$
\left|P R_{\nu}(t, x)\right|_{\infty} \leq \max \left\{\left\|R_{0, \nu}\right\|_{\infty, P}, e^{-\gamma}\left\|R_{0, \nu}\right\|_{\infty, P}\right\} \leq\left\|R_{0, \nu}\right\|_{\infty, P} .
$$

Next we proceed by induction on intervals of the form $t \in\left[n / c_{\min },(n+1) / c_{\min }\right]$ with $n \in \mathbb{N}$. Suppose that:

$$
\forall t \in\left[n / c_{\min },(n+1) / c_{\min }\right],\left\|R_{\nu}(t, \cdot)\right\|_{\infty, P} \leq e^{-\gamma n}\left\|R_{0, \nu}\right\|_{\infty, P} .
$$

Let $(n+1) / c_{\min } \leq t \leq(n+2) / c_{\min }$ and $x$ be in $[0,1]$. Constructing the light cone enclosed by lines with slopes $1 / c_{\min }$ and $1 / c_{\max }$ and passing through $(t, x)$, one gets the existence of a $t^{\star} \in\left[n / c_{\min },(n+2) / c_{\min }\right]$ such that:

$$
\left|P R_{\nu}(t, x)\right|_{\infty} \leq\left|P R_{\nu}\left(t^{\star}, 0\right)\right|_{\infty} \leq\left|P[H \cdot+B \sigma(K \cdot)] R_{\nu}\left(t^{\star}, 1\right)\right| .
$$

Using same reasoning as in the case $n=0$, it can be proved that:

$$
\left\|R_{\nu}\left(t^{\star}, \cdot\right)\right\|_{\infty, P} \leq\left\|R_{\nu}\left(n / c_{\min }, \cdot\right)\right\|_{\infty, P} .
$$

Hence, by the hypothesis of induction:

$$
\left|P R_{\nu}\left(t^{\star}, 1\right)\right|_{\infty} \leq\left\|R_{\nu}\left(t^{\star}, \cdot\right)\right\|_{\infty, P} \leq e^{-\gamma n}\left\|R_{0, \nu}\right\|_{\infty, P} \leq\left\|R_{0, \nu}\right\|_{\infty, P} .
$$

As a consequence, by (3.19):

$$
\left|P R_{\nu}\left(t^{\star}, 1\right)\right|_{\infty} \leq \frac{\left|P B P^{-1}\right|_{\infty} P_{\min } \sigma_{s}}{\left.|| P(H+B K) P^{-1}\right|_{\infty}+\left|P B P^{-1}\right|_{\infty}\left|P K P^{-1}\right|_{\infty}-e^{-\gamma} \mid}
$$

Thus, we can use Lemma 3.15 in (3.21) to get: 


$$
\begin{aligned}
\left|P R_{\nu}(t, x)\right|_{\infty} & \leq e^{-\gamma}\left|P R_{\nu}\left(t^{\star}, 1\right)\right|_{\infty} \\
& \leq e^{-\gamma}\left\|R_{\nu}\left(t^{\star}, \cdot\right)\right\|_{\infty, P} .
\end{aligned}
$$

Hence by the induction hypothesis,

$$
\left\|R_{\nu}(t, \cdot)\right\|_{\infty, P} \leq e^{-\gamma}\left\|R_{\nu}\left(t^{\star}, \cdot\right)\right\|_{\infty, P} \leq e^{-\gamma(n+1)}\left\|R_{0, \nu}\right\|_{\infty, P}
$$

where (3.22) has been used. To conclude, we have:

$$
\forall t \geq 0,\left\|R_{\nu}(t, \cdot)\right\|_{\infty, P} \leq \max \left\{1, e^{-\gamma\left(c_{\min } t-1\right)}\right\}\left\|R_{0, \nu}\right\|_{\infty, P} .
$$

It remains to prove the exponential decay for the solution $R$. It suffices to use property (2.3) and to take a sequence of initial data piecewise constant such that:

$$
\forall \nu>1,\left\|R_{0, \nu}\right\|_{\infty, P} \leq\left\|R_{0}\right\|_{\infty, P} .
$$

Owing this, one passes to the limit as $\nu$ goes to infinity to get:

$$
\forall t \geq 0,\|R(t, \cdot)\|_{\infty, P} \leq \max \left\{1, e^{-\gamma\left(c_{\min } t-1\right)}\right\}\left\|R_{0}\right\|_{\infty, P} .
$$

This ends the proof of Proposition 3.12.

4. Numerical results. Here, we study a numerical example with saturation and show the relevance of the estimation of the region of attraction (3.14).

4.1. Relevance of the estimation of the basin of attraction. In this section, an example of system of scalar conservation laws is analyzed for $d=2$ with saturated feedback control law with $\sigma_{s}=1$. Matrices are defined as follows.

$$
H=\left(\begin{array}{cc}
0 & 1.1 \\
1 & 0
\end{array}\right), B=I_{2}, K=\left(\begin{array}{cc}
0 & -0.1050 \\
-0.1045 & 0
\end{array}\right) .
$$

We take a nonlinear flux $f(R)=\Lambda R+0.2\left(\arctan \left(R_{1}\right), \arctan \left(R_{2}\right)\right)$ with

$$
\Lambda=\left(\begin{array}{cc}
1 & 0 \\
0 & \sqrt{2}
\end{array}\right)
$$

The open-loop system can be represented by the graph given in Figure 4:

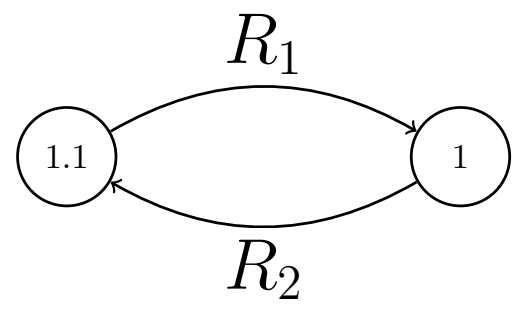

Figure 4: The open-loop system

We recall the estimation of the basin of attraction for $\gamma>0$ and $P \in D_{d}^{+}(\mathbb{R})$ :

$$
\left\|R_{0}\right\|_{\infty, P} \leq \frac{\left|P B P^{-1}\right|_{\infty} P_{\min } \sigma_{s}}{\left.|| P(H+B K) P^{-1}\right|_{\infty}+\left|P B P^{-1}\right|_{\infty}\left|P K P^{-1}\right|_{\infty}-e^{-\gamma} \mid} .
$$


Using an optimization routine from python, we calculate $P \in D_{d}^{+}(\mathbb{R})$ such that $\mid P(H+$ $B K)\left.P^{-1}\right|_{\infty}$ is minimal. The code gives:

$$
P=\left(\begin{array}{cc}
0.974 & 0 \\
0 & 1.026
\end{array}\right)
$$

To estimate the largest region of attraction, we take $\gamma=0$ in (4.1) which gives the following criteria of stability:

$$
\left\|R_{0}\right\|_{\infty, P} \leq \frac{\left|P B P^{-1}\right|_{\infty} P_{\min } \sigma_{s}}{\left.|| P(H+B K) P^{-1}\right|_{\infty}+\left|P B P^{-1}\right|_{\infty}\left|P K P^{-1}\right|_{\infty}-1 \mid} .
$$

4.2. Numerical simulations. Still keeping the matrices from previous section, we take a certain range of initial data $R_{0}$ constant on $[0,1]$ belonging to the estimated region of attraction and simulate the behavior of the solution. For example, one can take $R_{0}$ constant with value in $(-40,40)^{2}$ and look if the solution does not blow up at infinite time in $L^{\infty}$ norm. We briefly describe the scheme used. The space step is $d x=1 / N\left(N \in \mathbb{N}^{*}\right)$ and the time step $d t>0$ such that the following CFL condition holds:

$$
c_{\max } \frac{d t}{d x} \leq 1-\xi
$$

with

$$
0<\xi<1
$$

For computation, we take $d t=10^{-2}$ and $\frac{d t}{d x}=0.4$. Doing so, the space-time mesh is given by:

$$
\begin{aligned}
& \forall n \in \mathbb{N}, 1 \leq j \leq N,\left\{\begin{array}{l}
x_{j}:=(j-1 / 2) d x \\
t^{n}:=n d t .
\end{array}\right. \\
& \vdash_{x_{1}} x_{x_{2}} \ldots
\end{aligned}
$$

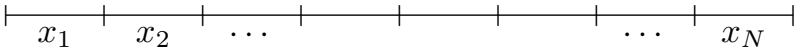

Figure 5: The space grid

The scheme is a finite volume one given by the minmod slope limiter method [24]. It is of the form:

$$
\frac{R_{j}^{n+1}-R_{j}^{n}}{d t}+\frac{f_{j+1 / 2}^{n}-f_{j-1 / 2}^{n}}{d x}=0
$$

where for all $n \geq 0,1 \leq i \leq 2$ :

$$
\begin{aligned}
\forall 2 \leq j \leq N-1, f_{i, j+1 / 2}^{n} & =f_{i}\left(R_{i, j}^{n}+\operatorname{minmod}\left(\frac{R_{i, j}^{n}-R_{i, j-1}^{n}}{d x}, \frac{R_{i, j+1}^{n}-R_{i, j}^{n}}{d x}\right) \frac{d x}{2}\right) \\
f_{i, N+1 / 2}^{n} & =f_{i}\left(R_{i, N}^{n}\right) \\
f_{i, N-1 / 2}^{n} & =f_{i}\left(R_{i, N-1}^{n}\right) \\
f_{i, 3 / 2}^{n} & =f_{i}\left(R_{i, 1}^{n}\right) \\
f_{i, 1 / 2}^{n} & =f_{i}\left(\left[(H+B \sigma(\cdot)) R_{N}^{n}\right]_{i}\right) .
\end{aligned}
$$


The minmod function is defined below for all $a, b \in \mathbb{R}$ :

$$
\operatorname{minmod}(a, b):= \begin{cases}0 & \text { if } a b \leq 0 \\ a & \text { if } a b \geq 0 \text { and }|a| \leq|b| \\ b & \text { otherwise. }\end{cases}
$$

One can cite [16] for the study of stability of such numerical system subject to linear boundary conditions.

In Figure 6, contours correspond to the rate of exponential decay wrt $L^{\infty}$ norm of the numerical solution for a time window of 50 seconds. If it is negative, the solution decays exponentially in norm. If it is positive, we have exponential divergence. The orange square is the estimated region of attraction while the blue one encloses the zone where saturation does not occur at $t=0$.

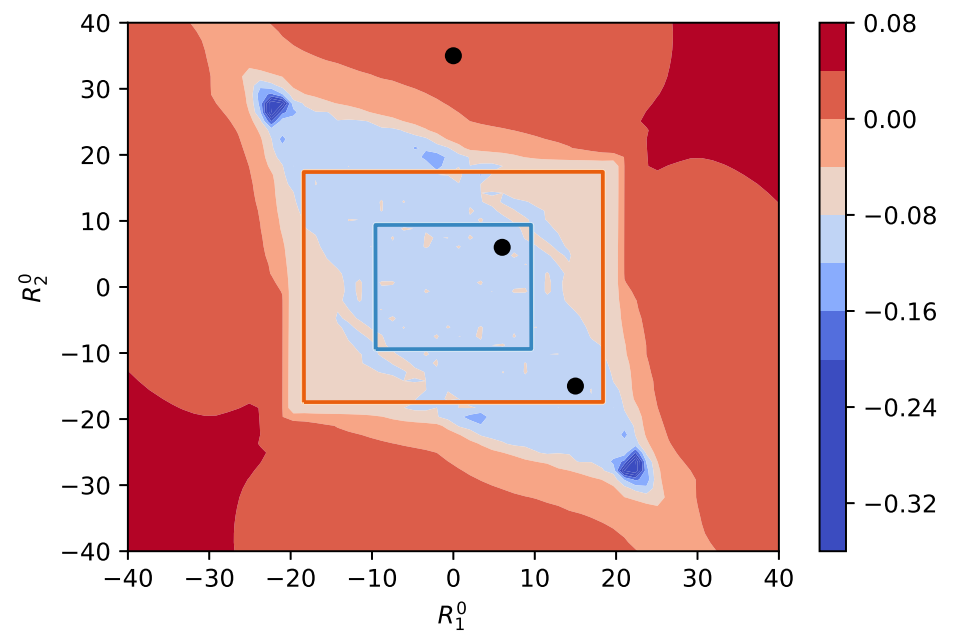

Figure 6: The basin of attraction

We also pick three initial data $R_{0}$ in different regions of Figure 6 and observe the dynamic of the solution. For example, one can take $R_{0}(x)=(15,-15)$ on $[0,1]$. The black dots in Figure 6 correspond to these initial data. The values of controls are plotted in Figure 7-9 where $u_{1}(t)=\sigma(K R(t, 1))_{1}$ and $u_{2}(t)=\sigma(K R(t, 1))_{2}$. Concerning Figure 7, we observe that some saturation occurs from $t=0$ until time $t \approx 13$, then the solution enters in the zone of linearity. The figure 8 represents a case where the system stays in the zone of linearity whereas in figure 9 , the initial data is out of the basin of attraction. 


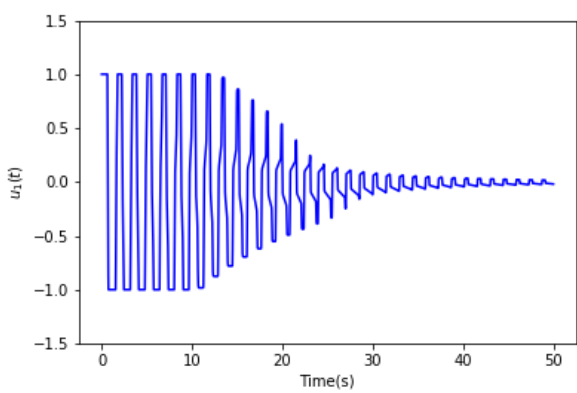

(a) The control $u_{1}(t)$

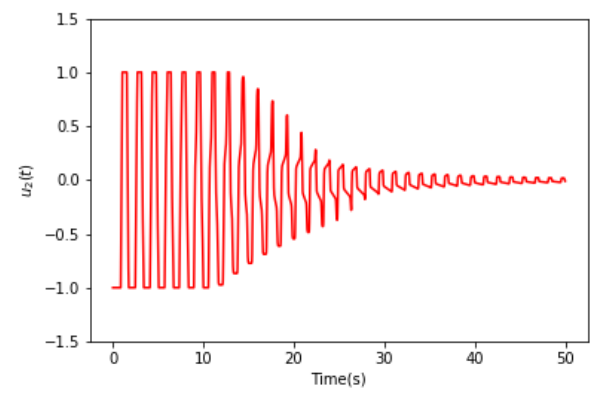

(b) The control $u_{2}(t)$

Figure 7: The case $R_{0}(x)=(15,-15)$

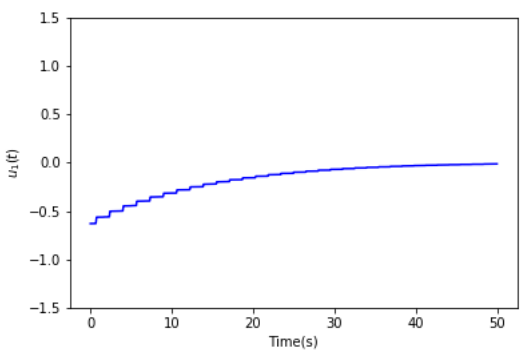

(a) The control $u_{1}(t)$

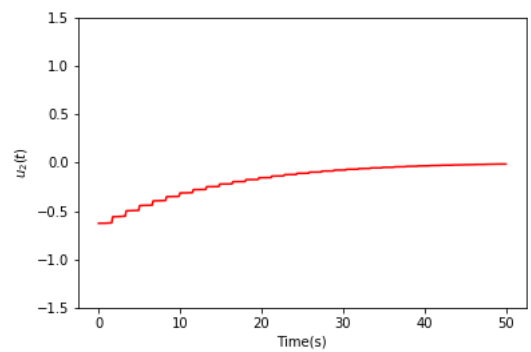

(b) The control $u_{2}(t)$

Figure 8: The case $R_{0}(x)=(6,6)$

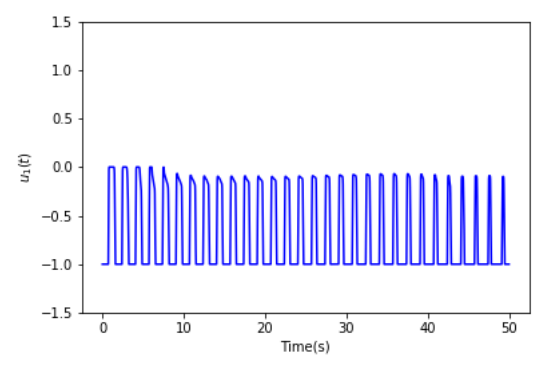

(a) The control $u_{1}(t)$

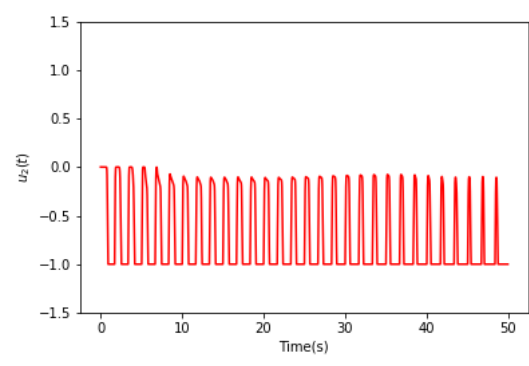

(b) The control $u_{2}(t)$

Figure 9: The case $R_{0}(x)=(0,35)$ 
5. Conclusion. The well-posedness for a wide class of systems of scalar conservation laws with boundary unsaturated and saturated feedback laws was established. The $\rho_{\infty}$ criteria was established in the $B V$ context for linear feedback laws. Then, for saturated feedback laws, we proved with an example that estimating a basin of attraction in $B V$ was not relevant. We rather gave an estimation of the basin of attraction in $L^{\infty}$ and deduce the exponential decay of the $B V$ norm of solutions whose initial data belongs to this basin of attraction.

Some questions remain open. The estimation (3.14) may not be optimal. Moreover, a method of maximizing the basin of attraction where the matrix $K$ is the variable of optimization is not given in this article. This is not an easy task since criterion (3.14) is not convex with respect to $K$. Finally, the other big gap to bridge is the stabilization of general systems of conservation laws the main difficulty coming from the well-posedness. The initial-boundary value problem for hyperbolic systems of conservation laws is indeed a very delicate matter, even when no characteristic speed vanishes. We refer to $[29,1,7,15,9]$ and the references therein.

Appendix A. Existence of a solution. All this section is dedicated to the proof of the existence result of Theorem 2.3.

A.1. The approximated problem. Let $\nu \geq 1, i$ in $\llbracket 1, d \rrbracket$ and define $f_{\nu, i}$ the piecewise affine approximation of $f_{i}$ coinciding with $f_{i}$ at all $2^{-\nu} j$ nodes $(j \in \mathbb{Z})$ by:

$f_{\nu, i}(s)=\frac{s-2^{-\nu} j}{2^{-\nu}} f_{i}\left(2^{-\nu}(j+1)\right)+\frac{2^{-\nu}(j+1)-s}{2^{-\nu}} f_{i}\left(2^{-\nu} j\right)$ for $s \in\left[2^{-\nu} j, 2^{-\nu}(j+1)\right]$.

The sequence $\left(f_{\nu}\right)_{\nu}$ is introduced in order to construct a piecewise constant entropy solution. The following lemma gives its main properties:

Lemma A.1. For all $T>0$, there exists a constant $C(g, T)$ such that for all $\nu>1$ and $R_{0, \nu}$ piecewise constant taking its values in $2^{-\nu} \mathbb{Z}$, there exists $R_{\nu}$ piecewise constant in the sense of Definition 2.1 verifying the following assertions:

- The approximated boundary condition (2.1) is verified.

- Two fronts cannot interact simultaneously with the right boundary.

- $\forall k \in \mathbb{R}^{d}, \phi \in C_{c}^{1}((0, T) \times(0,1) ; \mathbb{R})$ :

$$
\int_{0}^{T} \int_{0}^{1} \eta_{k}\left(R_{\nu}\right) \phi_{t}+q_{k}\left(R_{\nu}\right) \phi_{x} d x d t \geq-C(g, T) \frac{T V\left(R_{0, \nu}\right)}{\nu}\|\phi\|_{L^{\infty}\left(\mathbb{R}^{+} \times[0,1]\right)} .
$$

where :

$$
\left\{\begin{aligned}
\eta_{k}\left(R_{\nu}(t, x)\right) & =\sum_{i=1}^{d}\left|R_{\nu, i}(t, x)-k_{i}\right| \\
q_{k}\left(R_{\nu}(t, x)\right) & =\sum_{i=1}^{d}\left|f_{i}\left(R_{\nu, i}(t, x)\right)-f_{i}\left(k_{i}\right)\right| .
\end{aligned}\right.
$$

- The following bounds hold:

$$
\forall t \leq T, T V_{[0,1]}\left(R_{\nu}(t, .)\right) \leq C(g, T) T V_{[0,1]}\left(R_{0, \nu}\right) .
$$




$$
\forall t \leq T,\left\|R_{\nu}(t, .)\right\|_{L^{\infty}([0,1])} \leq C(g, T)\left\|R_{0, \nu}(t, .)\right\|_{L^{\infty}([0,1])} .
$$

Proof. See Appendix A.2

It is relatively easy to construct piecewise constant functions that make the entropy decrease. The main rules of construction are presented in the following lemma.

Lemma A.2 (Characterization of entropy piecewise constant functions).

$A$ piecewise constant $R$ in the sense of Definition 2.1 verifies the condition of entropy decay (1.14) if and only if for all integers $i$ in $\llbracket 1, d \rrbracket$ and all fronts $\gamma(t)$ of $R_{i}$,

- The Rankine-Hugoniot condition holds for $R_{i}^{l}$ the left state and $R_{i}^{r}$ the right state:

$$
\dot{\gamma}(t)\left[R_{i}^{r}(t)-R_{i}^{l}(t)\right]=f_{i}\left(R_{i}^{r}(t)\right)-f_{i}\left(R_{i}^{l}(t)\right) .
$$

- If $R_{i}^{l}(t)<R_{i}^{r}(t)$ then:

(A.5) $\forall \alpha \in[0,1], f_{i}\left(\alpha R_{i}^{r}(t)+(1-\alpha) R_{i}^{l}(t)\right) \geq \alpha f_{i}\left(R_{i}^{r}(t)\right)+(1-\alpha) f_{i}\left(R_{i}^{l}(t)\right)$.

- If $R_{i}^{l}(t)>R_{i}^{r}(t)$ then:

(A.6) $\forall \alpha \in[0,1], f_{i}\left(\alpha R_{i}^{r}(t)+(1-\alpha) R_{i}^{l}(t)\right) \leq \alpha f_{i}\left(R_{i}^{r}(t)\right)+(1-\alpha) f_{i}\left(R_{i}^{l}(t)\right)$.

Proof. This corresponds to [6, Theorem 4.4].

We call conditions (A.5)-(A.6), the entropy decay conditions of fronts; it selects values before and after the front such that the entropy of the solution decreases with time. If a front verifies such conditions, we say that the front is entropic.

A.2. Proof of Lemma A.1. Now we prove Lemma A.1 constructing step by step a piecewise constant solution. We begin by solving a Riemann problem to get a solution near $t=0$.

A.2.1. The Riemann problem. Let $i$ be an integer of $\llbracket 1, d \rrbracket, \nu>1$ and $R_{i}^{l}, R_{i}^{r} \in \mathbb{R}$ be two states. We recall techniques from [6, pp .108-113] to solve the Riemann problem associated to $\left(R_{i}^{l}, R_{i}^{r}\right)$ when taking $f_{\nu}$ as flux. There are two cases to consider:

- If $R_{i}^{l}<R_{i}^{r}$. Then, we consider $f_{i}^{*}$ the largest convex function inferior to $f_{\nu, i}$ on $\left[R_{i}^{r}, R_{i}^{l}\right]$. Denote also $w_{0}:=R_{i}^{l}<w_{1}<w_{2}<\cdots<w_{n}:=R_{i}^{r}$ the states where $f_{i}^{*, I}$ jumps. We give an example for $n=2$ on Figure 10: 


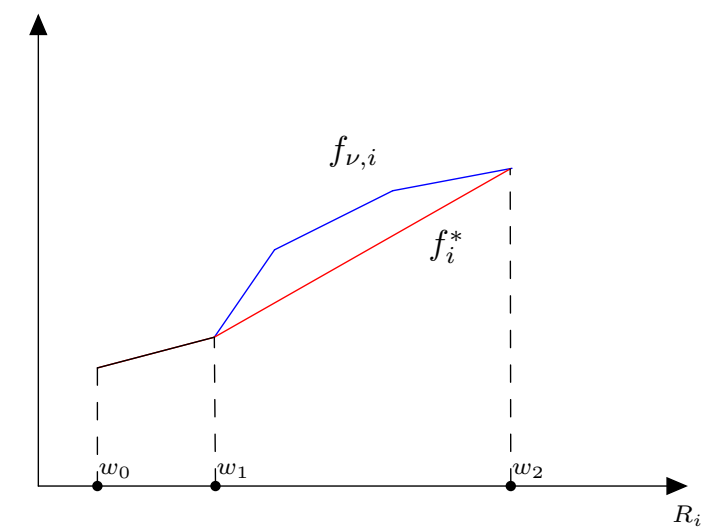

Figure 10: The case $R_{i}^{l}<R_{i}^{r}$

Introducing the speeds

$$
\lambda_{l}=\frac{f_{\nu, i}\left(w_{l}\right)-f_{\nu, i}\left(w_{l-1}\right)}{w_{l}-w_{l-1}}, l \in \llbracket 1, n \rrbracket,
$$

we define the solution to the Riemann problem as:

$$
R_{\nu, i}(t, x)=\left\{\begin{array}{c}
w_{0} \text { if } x<t \lambda_{1} \\
w_{l} \text { if } t \lambda_{l}<x<t \lambda_{l+1}, l \in \llbracket 1, n-1 \rrbracket \\
w_{n} \text { if } x>t \lambda_{n} .
\end{array}\right.
$$

This solution is entropic because it is piecewise constant, all fronts are entropic (A.5) and satisfy the Rankine-Hugoniot condition (A.4).

- If $R_{i}^{l}>R_{i}^{r}$. Then, we consider $f_{i}^{*}$ the smallest concave function larger than $f_{\nu, i}$. Denote also $w_{0}:=R_{i}^{l}>w_{1}>w_{2}>\cdots>w_{n}:=R_{i}^{r}$ the states where $f_{i}^{*, \prime}$ jumps. We give an example for $n=2$ on the Figure 11 .

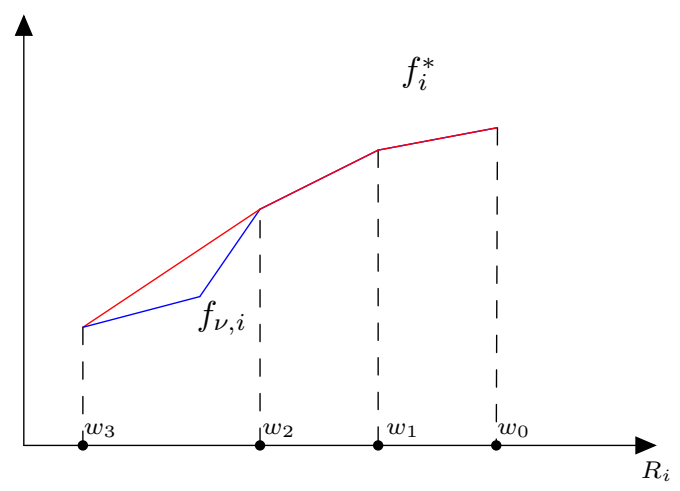

Figure 11: The case $R_{i}^{l}>R_{i}^{r}$ 
Defining velocities $\left(\lambda_{l}\right)_{l \in \llbracket 1, q \rrbracket}$ as in (A.7), we define the local solution also as:

$$
R_{\nu, i}(t, x)=\left\{\begin{array}{c}
w_{0} \text { if } x<t \lambda_{1} \\
w_{l} \text { if } t \lambda_{l}<x<t \lambda_{l+1}, l \in \llbracket 1, n-1 \rrbracket \\
w_{n} \text { if } x>t \lambda_{n} .
\end{array}\right.
$$

A.2.2. Local in time solution. Take a fixed $\nu>1$. Let us define what we will call the limit line $t \mapsto c_{\text {max }} t$ with maximal speed. Thanks to the Riemann solver defined in the previous section, we can find an entropy solution until a front interaction happens. The corresponding picture is given in Figure 12.

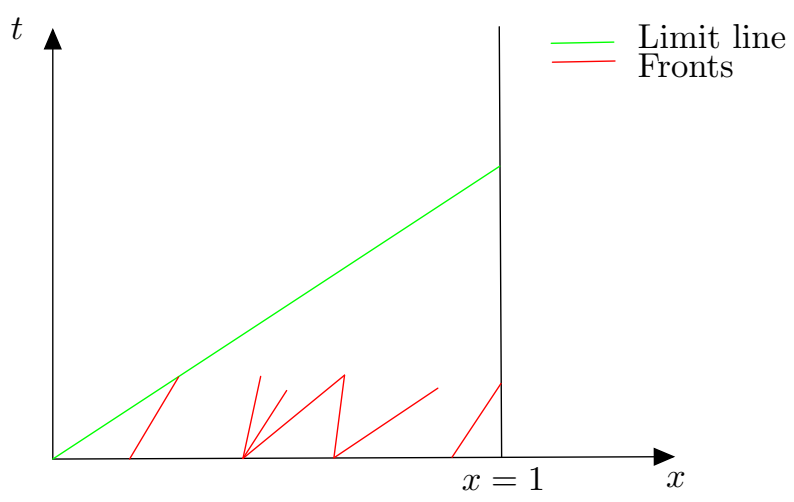

Figure 12: The local in time solution

A.2.3. Dealing with shock interactions. We recall the method described in [6, pp. 111-112]. Two cases have to be considered:

- (Case 1) All the incoming jumps have the same sign. Suppose they are all positive and let us denote $w_{0}<w_{1}<\ldots<w_{n}(n \in \mathbb{N})$ the consecutive "incoming" states. As all incoming fronts are entropic, we have:

$$
\forall \alpha \in[0,1], f\left(\alpha w_{i}+(1-\alpha) w_{i+1}\right) \geq \alpha f\left(w_{i}\right)+(1-\alpha) f\left(w_{i+1}\right) .
$$

The fact that we have converging fronts gives that the function $h$ built from lines passing through points $\left(w_{i}, f\left(w_{i}\right)\right)_{i \in \llbracket 0, n \rrbracket}$ is concave. Moreover, by (A.10):

$$
\forall w \in\left[w_{0}, w_{n}\right], h(w) \leq f(w) .
$$

Hence, by the concavity of $h$ and (A.11):

$\forall \alpha \in[0,1], f\left(\alpha w_{0}+(1-\alpha) w_{n}\right) \geq h\left(\alpha w_{0}+(1-\alpha) w_{n}\right) \geq \alpha f\left(w_{0}\right)+(1-\alpha) f\left(w_{n}\right)$.

Thus, it is possible to link the extremal $\left(w_{0}\right.$ and $\left.w_{n}\right)$ states by a unique entropic front whose jump intensity is strictly equal to the sum of the intensities 
of incoming jumps. Hence, in this case the total variation is conserved and so is the $L^{\infty}$ norm.
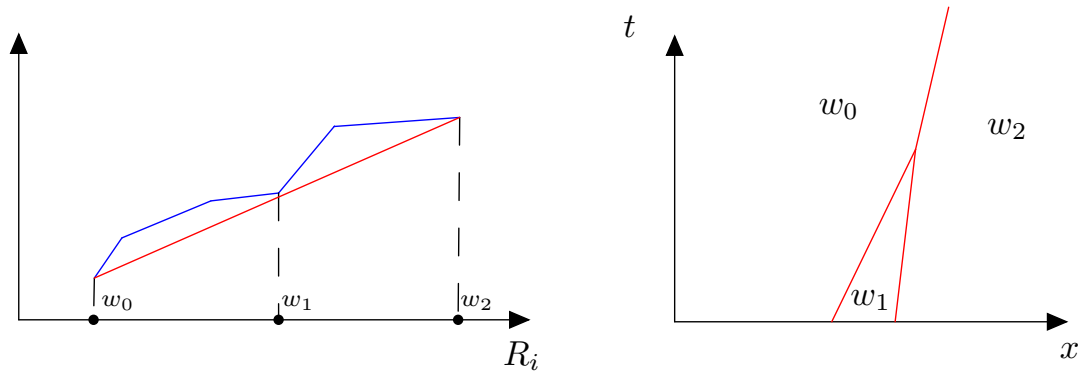

Figure 13: All jumps have the same sign

- (Case 2) Not all jumps have the same sign. Let us denote $w_{0}, w_{1}, \ldots, w_{n}$ $(n \in \mathbb{N})$ the consecutive "incoming" states. It is possible to link the extremal $\left(w_{0}\right.$ and $\left.w_{n}\right)$ states using fronts whose jumps have the same sign. To do so, it suffices to solve a Riemann problem between extremal states $w_{0}$ and $w_{n}$ as in Section A.2.1. Moreover, by the triangle inequality, the total variation decreases at least by $2 \times 2^{-\nu}$. Concerning the $L^{\infty}$ norm, it is conserved. This is because the $f_{\nu, i} \mathrm{~S}$ are non decreasing.
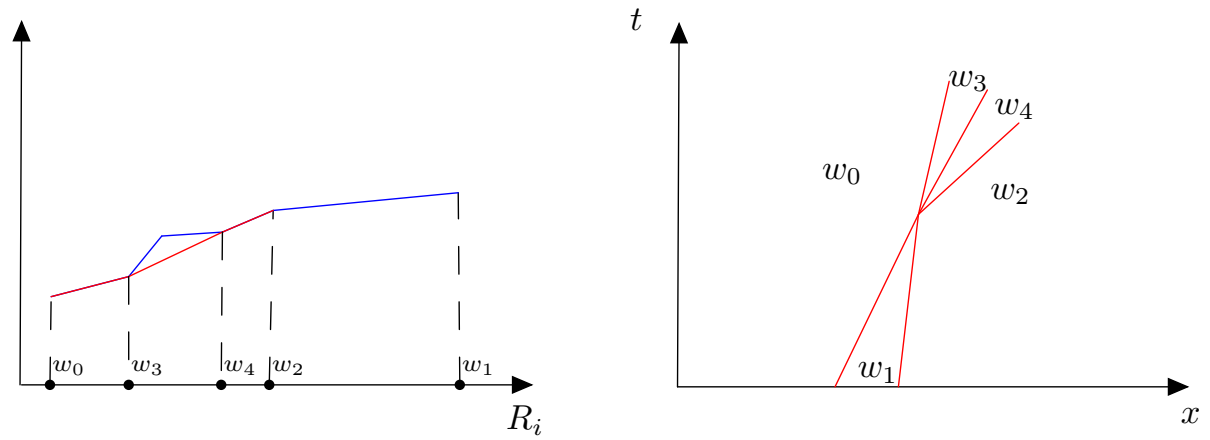

Figure 14: Not all jumps have the same sign

(Case 1) creates a unique front and let the total variation unchanged whereas (Case 2) can create several fronts but the total variation decreases by at least by $2 \times 2^{-\nu}$. Consequently, (Case 2) can happen only a finite number of times and the number of fronts remains bounded as time evolves. As a consequence, it is possible to construct an entropy piecewise constant approximate solution under the limit line verifying

$$
\begin{cases}\forall 0 \leq t \leq 1 / c_{\max }, T V_{\left[c_{\max } t, 1\right]}\left(R_{\nu, i}(t, .)\right) & \leq T V_{[0,1]}\left(R_{0, \nu, i}\right) \\ \forall x \in[0,1], T V_{\left[0, x / c_{\max }\right]}\left(R_{\nu, i}(., x)\right) & \leq T V_{[0,1]}\left(R_{0, \nu, i}\right)\end{cases}
$$


The corresponding picture is given in Figure 15:

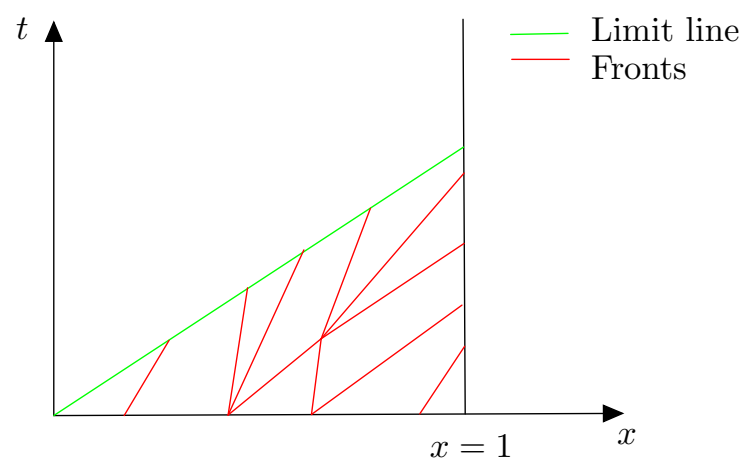

Figure 15: The solution under the limit line

Remark A.3. If $n \geq 2(n \in \mathbb{N})$ fronts interacts exactly at $x=1$ for some time $t>0$. Then, we modify a bit the velocity of $n-1$ fronts to prevent this situation. Taking one of such fronts, we denote $\lambda$ and $\tilde{\lambda}$ the respective former and new velocities. We can choose them such that $|\lambda-\tilde{\lambda}| \leq \frac{1}{\nu}$.

A.2.4. Finishing the construction. To construct locally the solution above the limit line, we impose the boundary condition

$$
\forall 0 \leq t \leq 1 / c_{\max }, R_{\nu}\left(t, 0^{+}\right)=g_{2 \nu}\left(R_{\nu}\left(t, 1^{-}\right)\right)
$$

where we recall that:

$$
\forall R \in \mathbb{R}^{d}, \nu>1, i \in \llbracket 1, d \rrbracket, g_{\nu, i}(R)=2^{-\nu}\left(E\left(2^{\nu} g_{i}(R)\right)\right) .
$$

Then, to construct a local solution, we solve the different Riemann problems as in section A.2.2 this time using the approximated flux $f_{2 \nu}$. More precisely if at a time $t$, $R_{i}\left(t^{-}, 0^{+}\right) \neq R_{i}\left(t^{+}, 0^{+}\right)$, we solve the Riemann problem with $R_{i}\left(t^{+}, 0^{+}\right)$as left state and $R_{i}\left(t^{-}, 0^{+}\right)$as right state. Hence we are able to get a solution locally above the limit line taking its values in $2^{-2 \nu} \mathbb{Z}$. 


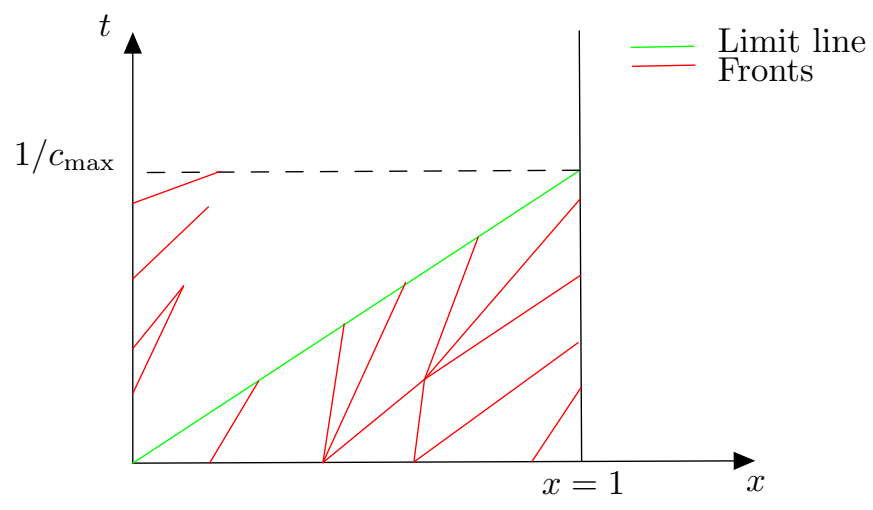

Figure 16: The local solution above the limit line

Finally, we extend fronts coming from the zone under the limit line and deal with front interactions as in section A.2.3 this time using the approximated flux $f_{2 \nu}$. The final picture is given in Figure 17. This is very important to remark that the picture under the limit line cannot be modified by fronts coming from the left boundary. This is because the limit line has maximal velocity.

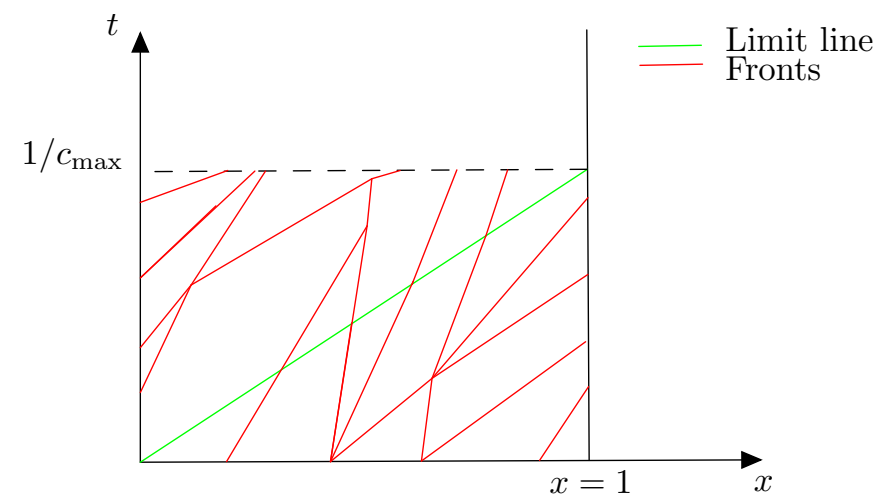

Figure 17: The solution

Concerning the total variation, the way we dealt with front interactions prevents the total variation from increasing when we compare the total strength of ongoing fronts with the one of outgoing fronts. As a consequence,

$$
\left\{\begin{aligned}
\forall 0 \leq t \leq 1 / c_{\max }, T V_{\left[0, c_{\max } t\right]}\left(R_{\nu, i}(t, .)\right) \leq & T V_{[0,1]}\left(R_{0, \nu, i}\right) \\
& +T V_{\left[0,1 / c_{\max }\right]}\left(R_{\nu, i}\left(., 0^{+}\right)\right) \\
\forall x \in[0,1], T V_{\left[x / c_{\max }, 1 / c_{\max }\right]}\left(R_{\nu, i}(., x)\right) \leq & T V_{[0,1]}\left(R_{0, \nu, i}\right) \\
& +T V_{\left[0,1 / c_{\max }\right]}\left(R_{\nu, i}\left(., 0^{+}\right)\right)
\end{aligned}\right.
$$


A.2.5. Conclusion. All previous steps can be repeated on intervals $\left[k / c_{\max },(k+1) / c_{\max }\right]$ and a solution defined for all time is built. Now let $T>0$. There are several points to verify:

- (Boundary condition) The approximated boundary condition (2.1) is satisfied by construction.

- (Boundary interactions) Two fronts cannot interact simultaneously at the right boundary by construction.

- (Estimate on the total variation). Using (A.12), (A.13) and the fact that $R_{\nu}$ satisfies the approximated boundary condition (2.1), one can deduce that there exists a constant $C(g, T)$ (depending on the Lipschitz constant of $g$ and T) such that

$$
\forall 0 \leq t \leq T, T V_{[0,1]}\left(R_{\nu}(t, .)\right) \leq C(g, T) T V_{[0,1]}\left(R_{0, \nu}(t, .)\right) .
$$

- (Estimate on the entropy) Take a positive test function $\phi \in C_{c}^{1}((0, T) \times(0,1))$, $T>0$ and $k \in \mathbb{R}^{d}$. Then, by integration by parts, one obtains:

$$
\int_{0}^{T} \int_{0}^{1}\left[\eta_{k}\left(R_{\nu}\right) \phi_{t}+q_{k}\left(R_{\nu}\right) \phi_{x} d x d t=\sum_{\substack{\left.\alpha \\\left(q_{k}\left(R_{\nu, \alpha}^{r}\right)-q_{k}\left(R_{\nu, \alpha}^{l}\right)\right)\right] \phi\left(t, \gamma_{\alpha}\right)}}\right.
$$

where $\alpha$ runs over the discontinuities $t \rightarrow\left(t, \gamma_{\alpha}(t)\right)$ of $R_{\nu}$.

We denote $P$ the set of physical fronts $i e$ the fronts for which the velocity has not been modified. $N P$ designates the complement of $P$. As fronts of $P$ are entropic by construction, we have:

$$
\begin{aligned}
& \int_{0}^{T} \int_{0}^{1} \eta_{k}\left(R_{\nu}\right) \phi_{i, t}+q_{k}\left(R_{\nu}\right) \phi_{i, x} d x d t \\
& \geq 0+\sum_{\alpha \in N P}\left[\dot{\gamma_{\alpha}}\left(\eta_{k}\left(R_{\nu, \alpha}^{r}\right) \eta_{k}\left(R_{\nu, \alpha}^{l}\right)\right)-\left(q_{k}\left(R_{\nu, \alpha}^{r}\right)-q_{k}\left(R_{\nu, \alpha}^{l}\right)\right)\right] \phi\left(t, \gamma_{\alpha}\right) \\
& =\sum_{\alpha \in N P}\left[\tilde{\lambda_{\alpha}}\left(\eta_{k}\left(R_{\nu, \alpha}^{r}\right)-\eta_{k}\left(R_{\nu, \alpha}^{l}\right)\right)-\left(q_{k}\left(R_{\nu, \alpha}^{r}\right)-q_{k}\left(R_{\nu, \alpha}^{l}\right)\right)\right] \phi\left(t, \gamma_{\alpha}\right)
\end{aligned}
$$

where we replaced the notation $\dot{\gamma}$ by $\tilde{\lambda}$ to emphasize the fact that it corresponds to a modified velocity (see Remark A.3); the unmodified "entropic" velocity being denoted $\lambda$. Hence,

$$
\begin{aligned}
& \int_{0}^{T} \int_{0}^{1} \eta_{k}\left(R_{\nu}\right) \phi_{t}+q_{k}\left(R_{\nu}\right) \phi_{x} d x d t \\
& \geq \sum_{\alpha \in N P}\left[\tilde{\lambda_{\alpha}}\left(\eta_{k}\left(R_{\nu, \alpha}^{r}\right)-\eta_{k}\left(R_{\nu, \alpha}^{l}\right)\right)-\left(q_{k}\left(R_{\nu, \alpha}^{r}\right)-q_{k}\left(R_{\nu, \alpha}^{l}\right)\right)\right] \phi\left(t, \gamma_{\alpha}\right) \\
& =\sum_{\alpha \in N P}\left[\lambda_{\alpha}\left(\eta_{k}\left(R_{\nu, \alpha}^{r}\right)-\eta_{k}\left(R_{\nu, \alpha}^{l}\right)\right)-\left(q_{k}\left(R_{\nu, \alpha}^{r}\right)-q_{k}\left(R_{\nu, \alpha}^{l}\right)\right)\right. \\
& \left.+\left(\tilde{\lambda_{\alpha}}-\lambda_{\alpha}\right)\left(\eta_{k}\left(R_{\nu, \alpha}^{r}\right)-\eta_{k}\left(R_{\nu, \alpha}^{l}\right)\right)\right] \phi\left(t, \gamma_{\alpha}\right) \\
& \geq 0-\frac{T V\left(R_{\nu}(t, .)\right)}{\nu}\|\phi\|_{L^{\infty}\left(\mathbb{R}^{+} \times[0,1]\right)} \\
& \geq-C(g, T) \frac{T V\left(R_{0, \nu}\right)}{\nu} \mid \phi \|_{L^{\infty}\left(\mathbb{R}^{+} \times[0,1]\right)}
\end{aligned}
$$

where we have used chronologically: 
- The fact that an unmodified velocity corresponds to an entropy front

- The equation $|\lambda-\tilde{\lambda}| \leq \frac{1}{\nu}$ from Remark A.3

- The fact that $\eta_{k}$ is 1 -Lipschitz

- The estimate (A.14) proven before.

- ( $L^{\infty}$ estimate). Remark that when we solved Riemann problem, the $L^{\infty}$ norm did not increase. This is mainly because we are dealing with non decreasing fluxes. The only way for the $L^{\infty}$ norm to increase is through the boundary condition. As a consequence, the estimate (A.3) holds.

This finishes the proof of Lemma A.1.

A.3. End of the proof of the existence result. To conclude on the existence, we will use Lemma A.1 and Helly's Theorem 1.4. There are several points to prove:

- (Entropy decay) Take $T>0, R_{0} \in B V([0,1])$ and a sequence $\left(R_{0, \nu}\right)_{\nu}$ of piecewise constant functions converging to $R_{0}$ in $B V$ (such a sequence exists by [6, Lemma 2.2]). For all $\nu>1$, we denote $\left(R_{\nu}\right)_{\nu}$ the sequence of piecewise constant functions of Lemma A.1.

By Lemma A.1, there exists a $C(g, T)>0$ such that

$$
\forall 0 \leq t \leq T, T V_{[0,1]}\left(R_{\nu}(t, .)\right) \leq C(g, T) T V_{[0,1]}\left(R_{0, \nu}\right) .
$$

As $\lim _{\nu \rightarrow \infty} R_{0, \nu}=R_{0} \in B V([0,1])$,

$$
\forall 0 \leq t \leq T, T V_{[0,1]}\left(R_{\nu}(t, .)\right) \leq C\left(g, T, R_{0}\right) .
$$

Next by (A.3) and the fact that the $L^{\infty}$ norm of the elements of $\left(R_{0, \nu}\right)_{\nu}$ are bounded, we have

$$
\forall 0 \leq t \leq T,\left\|R_{\nu}(t, .)\right\|_{L^{\infty}([0,1])} \leq C\left(g, T, R_{0}\right) .
$$

Finally for all $0 \leq s, t \leq T$ and by the finiteness of the speed of propagation:

$$
\begin{aligned}
\left\|R_{\nu}(t, .)-R_{\nu}(s, .)\right\|_{L^{1}([0,1])} & \leq c_{\max }(t-s) \max _{u \in[s, t]} T V_{[0,1]}\left(R_{\nu}(u, .)\right) \\
& \leq c_{\max }(t-s) C\left(g, T, R_{0}\right)
\end{aligned}
$$

where we have used (A.15).

By Helly's Theorem (Theorem 1.4), there exists a subsequence of $\left(R_{\nu}\right)_{\nu}$ still denoted $\left(R_{\nu}\right)_{\nu}$ converging in $L_{l o c}^{1}\left(\mathbb{R}^{+}, L^{1}([0,1])\right)$ to an element $R \in$ $L_{l o c}^{\infty}\left(\mathbb{R}^{+}, B V([0,1])\right)$. Moreover,

$$
\forall 0 \leq s, t \leq T,\|R(t, .)-R(s, .)\|_{L^{1}([0,1])} \leq c_{\max }(t-s) C\left(g, T, R_{0}\right) .
$$

As $\left(f_{\nu}\right)_{\nu}$ converges uniformly towards $f$ on bounded intervals, we can pass to the limit in (A.1) to get (1.14).

- (Initial condition). Let $\varepsilon>0$ and $s>0$ 


$$
\begin{aligned}
\left\|R(0, .)-R_{\nu}(0, .)\right\|_{L^{1}([0,1]) \leq} & \|R(0, .)-R(s, .)\|_{L^{1}([0,1])} \\
& +\left\|R(s, .)-R_{\nu}(s, .)\right\|_{L^{1}([0,1])} \\
& +\left\|R_{\nu}(s, .)-R_{\nu}(0, .)\right\|_{L^{1}([0,1])} \\
\leq & 2 C\left(g, R_{0}\right) s+\left\|R(s, .)-R_{\nu}(s, .)\right\|_{L^{1}([0,1])}
\end{aligned}
$$

where we have used (A.18).

Integrating with respect to $s$ on an interval $[0, t]$ for $0 \leq t \leq 1 / c_{\max }$, one gets

$$
\begin{aligned}
\left\|R(0, .)-R_{\nu}(0, .)\right\|_{L^{1}([0,1]) \leq} & C\left(g, R_{0}\right) t \\
& +\frac{1}{t} \int_{0}^{t}\left\|R(s, .)-R_{\nu}(s, .)\right\|_{L^{1}([0,1])} d s \\
\leq & C\left(g, R_{0}\right) t \\
& +\frac{1}{t} \int_{0}^{1 / c_{\max }}\left\|R(s, .)-R_{\nu}(s, .)\right\|_{L^{1}([0,1])} d s
\end{aligned}
$$

Taking $t=\frac{\varepsilon}{2 C\left(g, R_{0}\right)}$ and $\nu$ sufficiently large such that $\int_{0}^{1 / c_{\max }} \| R(s,)-$. $R_{\nu}(s,.) \|_{L^{1}([0,1])} d s \leq \frac{\varepsilon^{2}}{4 C\left(g, R_{0}\right)}$, one finally obtains:

$$
\left\|R(0, .)-R_{\nu}(0, .)\right\|_{L^{1}([0,1])} \leq \varepsilon .
$$

By the fact that $\left(R_{0, \nu}\right)_{\nu}$ converges towards $R_{0}$ in $L^{1}([0,1])$, we deduce that $R(0,)=.R_{0}$ in a $L^{1}$ sense and $R(0,)=.R_{0}$ almost everywhere.

Remark A.4. We can repeat the same procedure for any $t \geq 0$ and

$$
\forall t \geq 0, \lim _{\nu \rightarrow \infty}\left\|R_{\nu}(t, .)-R(t, .)\right\|_{L^{1}([0,1])}=0 .
$$

- (Boundary condition). For the boundary condition, it suffices to consider the variable $x$ as a time variable.

Using (A.12), (A.13) and the approximated boundary condition (2.1), one can easily prove that

$$
\forall x \in[0,1], T V_{\left[0,1 / c_{\max }\right]}\left(R_{\nu}(., x)\right) \leq C(g) T V_{[0,1]}\left(R_{0, \nu}(.)\right) .
$$

As $\left(R_{0, \nu}\right)_{\nu}$ is bounded in $B V$,

$$
\forall x \in[0,1], T V_{\left[0,1 / c_{\max }\right]}(R(., x)) \leq C\left(g, R_{0}\right) .
$$

Additionally, with (A.16) we get the $L^{\infty}$ estimate

$$
\forall \nu>1, \forall x \in[0,1],\left\|R_{\nu}(., x)\right\|_{L^{\infty}\left(\left[0,1 / c_{\max }\right]\right)} \leq C\left(g, R_{0}\right) .
$$

Finally, using (A.19) and recalling the definition $c_{\min }:=\min _{i} \alpha_{i}$ of front velocities, we have for $0 \leq x, y \leq 1$ :

$$
\forall \nu>1,\left\|R_{\nu}(., x)-R_{\nu}(., y)\right\|_{L^{1}\left(\left[0,1 / c_{\max }\right]\right)} \leq \frac{|x-y|}{c_{\min }} C\left(g, R_{0}\right) .
$$

By Helly's Theorem (Theorem 1.4), $\left(R_{\nu}\right)_{\nu}$ tends towards $R$ in $L_{\text {loc }}^{\infty}\left([0,1], L^{1}\left(\left[0,1 / c_{\mathrm{max}}\right]\right)\right)$. Using a similar argument as in the previous item 
of the proof, one shows that $R\left(., 0^{+}\right)=g\left(R\left(., 1^{-}\right)\right)$in the almost everywhere sense on $\left[0,1 / c_{\max }\right]$. We repeat the argument to get the same conclusion for all time.

Hence, $R$ is a solution of (1.13) in the sense of Definition 1.6. It remains to prove that $\left(R_{\nu}\right)_{\nu}$ is an approximating sequence of PCFs of the entropy solution $R$ in the sense of Definition 2.2. By construction, $\left(R_{\nu}\right)_{\nu}$ satisfies the first five points of Definition 2.2. It remains only to prove the bound

$$
\forall t \geq 0, \delta t>0, T V_{[0,1]}(R(t, .)) \leq \limsup _{\nu \rightarrow \infty} \sup _{s \in[t, t+\Delta t]} T V_{[0,1]}\left(R_{\nu}(s, .)\right) .
$$

This is a consequence of Helly's Theorem. Indeed, take $t \geq 0, \Delta t>0$ and $n \in \mathbb{N}^{*}$. Instead of applying Helly's Theorem on an interval of the form $[0, T]$ for the sequence $\left(R_{\nu}\right)_{\nu>1}$, we apply it on the interval $[t, t+\Delta t]$ for the sequence $\left(R_{\nu}\right)_{\nu>n}$.

As

$$
\forall s \in[t, t+\Delta t], \forall \nu>n, T V_{[0,1]}\left(R_{\nu}(s, .)\right) \leq \sup _{\substack{u \in[t, t+\Delta t] \\ v>n}} T V_{[0,1]}\left(R_{v}(u, .)\right)
$$

we deduce by Helly's Theorem that

$$
\forall s \in[t, t+\Delta t], T V_{[0,1]}(R(s, .)) \leq \sup _{\substack{u \in[t, t+\Delta t] \\ v>n}} T V_{[0,1]}\left(R_{v}(u, .)\right)
$$

Passing to the limit as $n$ goes to infinity gives (A.22). To get the estimate (2.3), the proof is similar. The existence part of Theorem 2.3 is proven.

Appendix B. Uniqueness. We will adapt the method of doubling variables of Kruzhkov to our boundary value problem. Let $u, v$ be two entropy solutions of (1.13) with their respective initial data $u_{0}, v_{0}$.

We will first show the uniqueness on the triangle $T_{1}$ :

$$
T_{1}:=\left\{(t, x) \mid c_{\max } t \leq x \leq 1,0 \leq t \leq 1 / c_{\max }\right\}
$$

To do so, let $0<t \leq 1 / c_{\max }$ and define the domain $\Omega_{t}$ by:

$$
\Omega_{t}:=\left\{(s, x) ; 0 \leq s \leq t, c_{\max } s \leq x \leq 1\right\}
$$

We give a graphical representation of $\Omega_{t}$ in Figure 18 . 


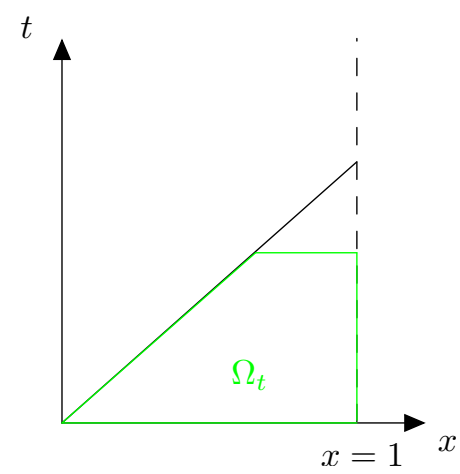

Figure 18: The domain $\Omega_{t}$

Formally, as $u$ in entropy on $\Omega_{t}$, we have for all $k \in \mathbb{R}^{d}$

$$
\begin{aligned}
0 \geq & \iint_{\Omega_{t}} \eta_{k}(u)_{t}+q_{k}(u)_{x} d x d t \\
= & \int_{1-c_{\max } t}^{1} \eta_{k}(u) d x-\int_{0}^{1} \eta_{k}\left(u_{0}\right) d x \\
& +\int_{0}^{t} c_{\max } \eta_{k}\left(u\left(\tau, c_{\max } \tau\right)\right)-q_{k}\left(u\left(\tau, c_{\max } \tau\right)\right) d \tau+\int_{0}^{t} q_{k}(u(1, s)) d s .
\end{aligned}
$$

The third term is positive because $c_{\max }$ is superior to all the Lipschitz constants of the $f_{i}$ s. The last term is positive since all the $f_{i}$ s are non decreasing. Hence,

$$
\int_{1-c_{\max } t}^{1} \eta_{k}(u) d x \leq \int_{0}^{1} \eta_{k}\left(u_{0}\right) d x
$$

It is equivalent to:

$$
\forall k \in \mathbb{R}^{d}, \sum_{i=1}^{d} \int_{1-c_{\max } t}^{1}\left|u_{i}(t, x)-k_{i}\right| d x \leq \sum_{i=1}^{d} \int_{0}^{1}\left|u_{0, i}(x)-k_{i}\right| d x .
$$

Kruzhkov's doubling variable method allows to replace the $k_{i}$ by the $v_{i}$ to give:

$$
\sum_{i=1}^{d} \int_{1-c_{\max } t}^{1}\left|u_{i}(t, x)-v_{i}(t, x)\right| d x \leq \sum_{i=1}^{d} \int_{0}^{1}\left|u_{0, i}(x)-v_{0, i}(x)\right| d x .
$$

Remark B.1. Rigorous justifications of previous computations can be found in the proof of [6, Theorem 6.2].

As a consequence, the solution is unique on the triangle $T_{1}$.

Now let $x$ be in ]0,1[, we apply the same strategy to the set

$$
\Omega_{x}:=\left\{(s, y) ; \quad 0 \leq y \leq x, y / c_{\max } \leq s \leq 1 / c_{\max }\right\}
$$

represented in Figure 19. 


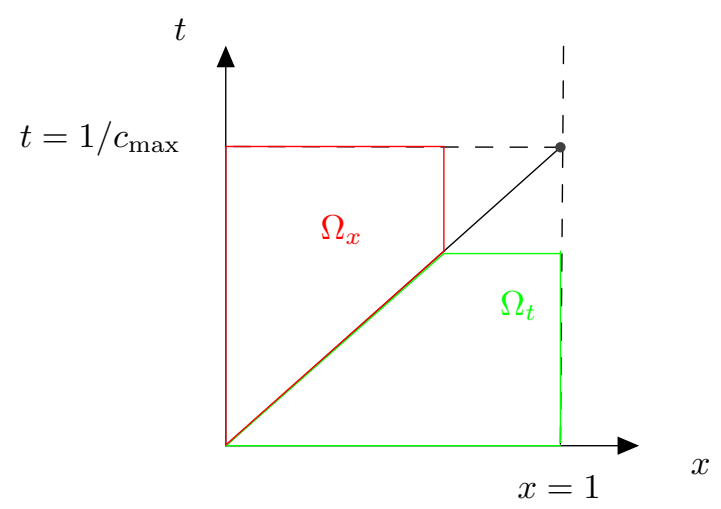

Figure 19: The domain $\Omega_{x}$

Integrating $\eta_{k}(u)_{t}+q_{k}(u)_{x} \leq 0$ in $\Omega_{x}$, one obtains: $\forall x \in[0,1], k \in \mathbb{R}^{d}$,

$$
\begin{aligned}
\int_{1 / c_{\max }-x / c_{\max }}^{1 / c_{\max }} q_{k}(u(t, x)) d t \leq & \int_{0}^{1 / c_{\max }} q_{k}(u(t, 0)) d t \\
& +\int_{0}^{x / c_{\max }} c_{\max } \eta_{k}\left(u\left(t, c_{\max } t\right)\right)-q_{k}\left(u\left(t, c_{\max } t\right)\right) d t .
\end{aligned}
$$

This is equivalent to: $\forall x \in[0,1], k \in \mathbb{R}^{d}$,

$$
\begin{aligned}
& \sum_{i=1}^{d} \int_{1 / c_{\max }-x / c_{\max }}^{1 / c_{\max }}\left(f_{i}\left(u_{i}\right)-f_{i}\left(k_{i}\right)\right) \operatorname{sign}\left(u_{i}-k_{i}\right) d t \\
& \leq \sum_{i=1}^{d} \int_{0}^{1 / c_{\max }}\left(f_{i}\left(u_{i}(t, 0)\right)-f_{i}\left(k_{i}\right)\right) \operatorname{sign}\left(u_{i}(t, 0)-k_{i}\right) d t \\
& +\int_{0}^{x / c_{\max }} c_{\max } \eta_{k}\left(u\left(t, c_{\max } t\right)\right)-q_{k}\left(u\left(t, c_{\max } t\right)\right) d t .
\end{aligned}
$$

As all the $f_{i}$ are non decreasing $\left(q_{k} \geq 0\right)$ and all $c_{\max }$ Lipschitz: $\forall x \in[0,1], k \in \mathbb{R}^{d}$,

$$
\begin{aligned}
\sum_{i=1}^{d} \int_{1 / c_{\max }-x / c_{\max }}^{1 / c_{\max }}\left|f_{i}\left(u_{i}\right)-f_{i}\left(k_{i}\right)\right| d t \leq & c_{\max } \sum_{i=1}^{d} \int_{0}^{1 / c_{\max }}\left|u_{i}(t, 0)-k_{i}\right| d t \\
& +c_{\max } \int_{0}^{x / c_{\max }} \eta_{k}\left(u\left(t, c_{\max } t\right)\right) d t
\end{aligned}
$$

Following Kruzhkov's method, the $k_{i} \mathrm{~s}$ can be replaced by the $v_{i}$ s and for all $x$ in $[0,1]$ :

$$
\begin{aligned}
\sum_{i=1}^{d} \int_{1 / c_{\max }-x / c_{\max }}^{1 / c_{\max }}\left|f_{i}\left(u_{i}\right)-f_{i}\left(v_{i}\right)\right| d t & \leq c_{\max } \sum_{i=1}^{d} \int_{0}^{1 / c_{\max }}\left|u_{i}(t, 0)-v_{i}(t, 0)\right| d t \\
& +c_{\max } \int_{0}^{x / c_{\max }}\left|u\left(t, c_{\max } t\right)-v\left(t, c_{\max } t\right)\right| d t .
\end{aligned}
$$


As $u, v$ satisfy the boundary condition on $\left[0,1 / c_{\max }\right]$, there exists a constant $C\left(g, c_{\max }\right)$ depending on the Lipschitz constant of $g$ such that for all $x$ in $[0,1]$ :

$$
\begin{aligned}
& \sum_{i=1}^{d} \int_{1 / c_{\max }-x / c_{\max }}^{1 / c_{\max }}\left|f_{i}\left(u_{i}\right)-f_{i}\left(v_{i}\right)\right| d t \\
& \leq C\left(g, c_{\max }\right) \sum_{i=1}^{d} \int_{0}^{1 / c_{\max }}\left|u_{i}(t, 1)-v_{i}(t, 1)\right| d t \\
& +c_{\max } \int_{0}^{x / c_{\max }}\left|u\left(t, c_{\max } t\right)-v\left(t, c_{\max } t\right)\right| d t .
\end{aligned}
$$

If $u_{0}=v_{0}$, we have seen that $u$ and $v$ coincide on $T_{1}$. This implies that if $u_{0}=v_{0}$, $u$ and $v$ coincide on the segment $\{1\} \times\left[0,1 / c_{\max }\right]$ and on the line $\left(x=c_{\max } t, t\right)$ for $t \leq 1 / c_{\max }$. As a consequence,

$$
\forall x \in[0,1], \sum_{i=1}^{d} \int_{1 / c_{\max }-x / c_{\max }}^{1 / c_{\max }}\left|f_{i}\left(u_{i}\right)-f_{i}\left(v_{i}\right)\right| d t=0 .
$$

By the monoticity of the $f_{i} \mathrm{~s}, u$ and $v$ coincide on the triangle $T_{2}$ defined by

$$
T_{2}:=\left\{(t, x) \mid 0 \leq x \leq 1, x / c_{\max } \leq t \leq 1 / c_{\max }\right\} .
$$

To conclude, $u$ and $v$ coincide for $t \leq 1 / c_{\max }$ and repeating this argument, we can prove the uniqueness for all time. This finishes the proof of the uniqueness.

Acknowledgments. I would like to sincerely thank F. Boyer, F. Ferrante and C. Prieur for our fruitful discussions.

\section{REFERENCES}

[1] D. Amadori and R. Colombo, Continuous dependence for $2 \times 2$ conservation laws with boundary, Journal of Differential Equations, 138 (1997), pp. 229 - 266, https:// doi.org/https://doi.org/10.1006/jdeq.1997.3274, http://www.sciencedirect.com/science/ article/pii/S0022039697932745.

[2] A. Aw And M. Rascle, Resurrection of "second order" models of traffic flow, SIAM J. Appl. Math., 60 (2000), pp. 916-938, https://doi.org/10.1137/S0036139997332099, https://doi. org/10.1137/S0036139997332099.

[3] G. Bastin and J.-M. Coron, Stability And Boundary Stabilization Of 1-D Hyperbolic Systems, Progress in Nonlinear Differential Equations and Their Applications, Springer International Publishing, 2016.

[4] G. Bastin, J.-M. Coron, And B. D'Andréa Novel, On Lyapunov stability of linearised SaintVenant equations for a sloping channel, Netw. Heterog. Media, 4 (2009), pp. 177-187, https://doi.org/10.3934/nhm.2009.4.177, https://doi.org/10.3934/nhm.2009.4.177.

[5] P. Bernard And M. Krstic, Adaptive output-feedback stabilization of non-local hyperbolic PDEs, Automatica J. IFAC, 50 (2014), pp. 2692-2699, https://doi.org/10.1016/j. automatica.2014.09.001, https://doi.org/10.1016/j.automatica.2014.09.001.

[6] A. Bressan, Hyperbolic Systems of Conservation Laws: The One-dimensional Cauchy Problem, Oxford Lecture Series in Mathematics, Oxford University Press, 2000.

[7] R. Colombo and G. Guerra, On general balance laws with boundary, Journal of Differential Equations, 248 (2010), pp. 1017 - 1043, https://doi.org/https://doi.org/10.1016/j.jde. 2009.12.002, http://www.sciencedirect.com/science/article/pii/S0022039609004550.

[8] J.-M. Coron, B. D'Andréa Novel, And G. Bastin, A strict Lyapunov function for boundary control of hyperbolic systems of conservation laws, IEEE Transactions on Automatic Control, 52 (2004), pp. 2-11.

[9] J.-M. Coron, S. Ervedoza, S. Ghoshal, O. Glass, and V. Perrollaz, Dissipative boundary conditions for $2 \times 2$ hyperbolic systems of conservation laws for entropy solutions in bv, Journal of Differential Equations, 262 (2017), pp. 1 - 30, https://doi.org/ https://doi.org/10.1016/j.jde.2016.09.016, http://www.sciencedirect.com/science/article/ pii/S0022039616302832. 
[10] J.-M. Coron And H. NGuYen, Dissipative boundary conditions for nonlinear 1-D hyperbolic systems: sharp conditions through an approach via time-delay systems, SIAM Journal on Mathematical Analysis, 47 (2015), pp. 2220-2240, https://doi.org/10.1137/140976625.

[11] J.-M. Coron AND Z. WANG, Output feedback stabilization for a scalar conservation law with a nonlocal velocity, SIAM Journal on Mathematical Analysis, 45 (2013), pp. 2646-2665, https://doi.org/10.1137/120902203.

[12] C. Dafermos, Hyperbolic conservation laws in continuum physics, Grundlehren Math. Wissenschaften Series, Vol 325, Springer Verlag, Third Edition, (2010).

[13] J. de Halleux, C. Prieur, J.-M. Coron, B. Novel, and G. Bastin, Boundary feedback control in networks of open channels, Automatica, 39 (2003), pp. 1365-1376, https://doi. org/10.1016/S0005-1098(03)00109-2.

[14] A. Diagne, G. Bastin, And J.-M. Coron, Lyapunov exponential stability of 1-D linear hyperbolic systems of balance laws, Automatica J. IFAC, 48 (2012), pp. 109-114, https://doi. org/10.1016/j.automatica.2011.09.030, https://doi.org/10.1016/j.automatica.2011.09.030.

[15] C. Donadello and A. Marson, Stability of front tracking solutions to the initial and boundary value problem for systems of conservation laws, Nonlinear Differential Equations and Applications NoDEA, 14 (2007), pp. 569-592, https://doi.org/10.1007/s00030-007-5010-7, https://doi.org/10.1007/s00030-007-5010-7.

[16] M. Dus, Exponential stability of a general slope limiter scheme for scalar conservation laws subject to a dissipative boundary condition. Preprint, Jan. 2021, https://hal.archives-ouvertes. $\mathrm{fr} /$ hal-03116551.

[17] M. Dus, F. Ferrante, And C. Prieur, On $L^{2}$ stabilization of diagonal semilinear hyperbolic systems by saturated boundary control, Submitted, (2019).

[18] J. Glimm, Solutions in the large for nonlinear hyperbolic systems of equations, Communications on Pure and Applied Mathematics, 18 (1965), pp. 697-715, https://doi.org/10.1002/ cpa.3160180408, https://onlinelibrary.wiley.com/doi/abs/10.1002/cpa.3160180408, https: //arxiv.org/abs/https://onlinelibrary.wiley.com/doi/pdf/10.1002/cpa.3160180408.

[19] J. K. Hale and M. Verduyn Lunel, Introduction To Functional Differential Equations, vol. 99 of Applied Mathematical Sciences, Springer Verlag, 1993.

[20] A. HAYAT, Exponential stability of general 1-D quasilinear systems with source terms for the $C^{1}$ norm under boundary conditions, Preprint, (2017).

[21] A. HAYAT, On boundary stability of inhomogeneous $2 \times 2$ 1-D hyperbolic systems for the $C^{1}$ norm, ESAIM Control Optim. Calc. Var., 25 (2019), pp. Paper No. 82, 31, https://doi. org/10.1051/cocv/2018059, https://doi.org/10.1051/cocv/2018059.

[22] R. J. DiPerna, Global existence of solutions to nonlinear hyperbolic systems of conservation laws, Journal of Differential Equations, 20 (1976), https://doi.org/10.1016/0022-0396(76) 90102-9.

[23] P. Lax, Hyperbolic Systems of Conservation Laws, Communications on Pure and Applied Mathematics, 10 (1957), pp. 537-566.

[24] R. J. LeVeque, Finite volume methods for hyperbolic problems, Cambridge Texts in Applied Mathematics, Cambridge University Press, Cambridge, 2002, https://doi.org/10.1017/ CBO9780511791253, https://doi.org/10.1017/CBO9780511791253.

[25] T. T. LI, Global classical solutions for quasilinear hyperbolic systems, RAM: Research in Applied Mathematics, 32 (1994).

[26] V. Perrolaz, Asymptotic stabilization of entropy solutions to scalar conservation laws through a stationary feedback law, Annales de l'Institut Henri Poincare (C) Non Linear Analysis, 30 (2013), pp. 879 - 915, https://doi.org/https://doi.org/10.1016/j.anihpc.2012.12.003, http: //www.sciencedirect.com/science/article/pii/S0294144913000036.

[27] C. Prieur, S. Tarbouriech, and J. M. Gomes da Silva Jr., Wave equation with cone-bounded control laws, IEEE Transactions on Automatic Control, 61 (2016), pp. 3452-3463.

[28] T. H. QIN, Global smooth solutions of dissipative boundary value problems for first order quasilinear hyperbolic systems, Chinese Ann. Math. Ser. B, 6 (1985), pp. $289-298$.

[29] M. Sablé-Tougeron, Méthode de Glimm et problème mixte, Annales de l'I.H.P. Analyse non linéaire, 10 (1993), pp. 423-443, http://www.numdam.org/item/AIHPC_1993__10_4_423_0.

[30] S. Tarbouriech, G. Garcia, J. M. Gomes da Silva Jr., and I. Queinnec, Stability and Stabilization of Linear Systems with Saturating Actuators, Springer London, 2011. 